\title{
A second order approximation for quasilinear non-Fickian diffusion models*
}

\author{
J.A. Ferreira $\dagger^{\dagger}$ E. Gudino and P. de Oliveira \\ CMUC, Department of Mathematics, University of Coimbra \\ 3001-454 Coimbra, Portugal
}

\begin{abstract}
In this paper initial boundary value problems, defined using quasilinear diffusion equations of Volterra type, are considered. These equations arise for instance to describe diffusion processes in viscoelastic media whose behaviour is represented by a Voigt-Kelvin model or a Maxwell model.

A finite difference discretization defined on a general nonuniform grid with second order convergence order in space is proposed. The analysis does not follow the usual splitting of the global error using the solution of an elliptic equation induced by the integro-differential equation. The new approach enables us to reduce the smoothness required to the theoretical solution when the usual split technique is used. Non singular and singular kernels are considered. Numerical simulation which shows the effectiveness of the method are included.
\end{abstract}

2010 Mathematical subject classification: 65M06;65M12

Keywords: Integro-differential equations of Volterra type, non-Fickian diffusion models

\section{Introduction}

When a fluid penetrates a viscoelastic material its transport is not accurately described by a classical diffusion-reaction equation. Brownian motion of fluid molecules should be connected by a term representing the stress response of the material to the deformation of the incoming fluid ([11], [12], [13], [14] and [38]). The fact that classical diffusion does not accurately describes transport phenomena is felt not only in polymer sciences but also in other scientific domains of material sciences ([28], [32] and [36]) as well as in life sciences ([19], [20], [25], [29] and [35]). To improve the mathematical description of such phenomena we consider the class of quasilinear integro-differential equations of Volterra type

$$
\begin{aligned}
& \frac{\partial c}{\partial t}(x, t)=\frac{\partial}{\partial x}\left(a(c(x, t)) \frac{\partial c}{\partial x}(x, t)\right)+\int_{0}^{t} k_{e r}(t-s) \frac{\partial}{\partial x}\left(d(c(x, s)) \frac{\partial c}{\partial x}(x, s)\right) d s+f \\
& \text { in }(0,1) \times(0, T],
\end{aligned}
$$

where $k_{e r}$ is a kernel function. In (1) $c$ represents a concentration, $a(c)$ stands for the diffusion coefficient, $d(c)$ for a viscoelastic diffusion coefficient and $f$ represents a reaction term. Equation (1) is completed with Dirichlet boundary conditions

$$
\begin{aligned}
& c(0, t)=c_{\text {in }}, \text { for } t \in(0, T], \\
& c(1, t)=c_{\text {out }}, \text { for } t \in(0, T],
\end{aligned}
$$

*Dedicated to Rolf Dieter Grigorieff on occasion of his 75th birthday

$\dagger$ ferreira@mat.uc.pt 
and initial condition

$$
c(x, 0)=c_{0}(x), x \in(0,1) .
$$

Equation (1) is usually used to replace the classical diffusion-reaction equation

$$
\frac{\partial c}{\partial t}=\frac{\partial}{\partial x}\left(a(c) \frac{\partial c}{\partial x}\right)+f \text { in }(0,1) \times(0, T]
$$

when Fick's law for the mass flux $J_{F}$,

$$
J_{F}(x, t)=-a(c(x, t)) \frac{\partial c}{\partial x}(x, t)
$$

does not accurately describes transport.

In equation (1) the mass flux is split into a Fickian contribution and a non Fickian one that is

$$
J=J_{F}+J_{n F}
$$

with $J_{F}$ given by (6) and

$$
J_{n F}(x, t)=-\int_{0}^{t} k_{e r}(t-s) d(c(x, s)) \frac{\partial c}{\partial x}(x, s) d s .
$$

Our aim is to generalize the results obtained in [5] and [24] for the linear version of the quasilinear equation (1) considered, for instance, in [1], [2], [10], [22] and [23], avoiding the use of an elliptic auxiliary problem induced by this equation. The paper is organized as follows. In Section 2 we introduce the spatial discretization using the piecewise linear finite element method. Its convergence is analysed in Section 3. In the main results of the paper, Theorems 1 and 2, we prove that a discrete $L^{2}$ norm of the spatial discretization error and of its discrete gradient are of second order in space for non singular kernels. The version of these results are also considered for weakly singular kernels. We stress that the kernel does not need to be of positive type as is often the case in the analysis presented for instance in [33] and [34].

We point out that the convergence analysis presented here does not use the approach introduced by Wheeler in [37] and largely followed in the literature. This approach is essentially based on the splitting of the spatial discretization error considering an elliptic problem induced by (1). In Section 4 we study fully discrete implicit-explicit methods for non singular and weakly singular kernels. The same convergence orders are established. In Section 5 we present a numerical illustration of our main convergence results -Theorems 1 and 2. Finally, some conclusions are included in Section 6.

\section{Finite difference method}

The finite difference method is introduced in what follows by considering a variational problem associated with the integro-differential equation (1). Without loss of generality we will consider homogenous Dirichlet boundary conditions. By $L^{2}(0,1), H_{0}^{1}(0,1)$ and $W^{s, \infty}(0,1), s=0,1$, we denote the usual Sobolev spaces where we consider the usual norms $\|\|,.\|.\|_{1}$ and $\|.\|_{s, \infty}$, respectively, being the two first norms induced by the usual inner products $(.,$.$) and (., .)_{1}$, respectively.

Let $v:[0,1] \times[0, T] \rightarrow$ be given. Then we associate with $v$ the vector valued function $\tilde{v}$ that maps $[0, T]$ into the set of all mappings of $[0,1]$ into $\mathbb{R}$ with $[\tilde{v}(t)](x)=v(x, t)$. In what follow we will omit the tilde to denote this function. 
Let $V$ be a Banach space. For $s=0,1$, by $\mathscr{C}^{s}([0, T], V)$ we denote the space of functions $v:[0, T] \mapsto V$ such that $v^{(s)}:[0, T] \mapsto V$ is continuous and

$$
\|v\|_{\mathscr{C}^{s}([0, T], V)}=\max _{t \in[0, T]}\left\|v^{(s)}(t)\right\|_{V}<\infty .
$$

Let $L^{2}(0, T, V)$ be the space of Bochner-measurable functions $v:(0, T) \mapsto V$ such that

$$
\|v\|_{L^{2}(0, T, V)}=\int_{0}^{T}\|v(t)\|_{V}^{2} d t<\infty .
$$

By $H^{s}(0, T, V)$ we denote the space of functions $v$ in $L^{2}(0, T, V)$ whose distributional time derivatives up to order $s$ are also in $L^{2}(0, T, V)$. In this space we consider the following norm

$$
\|v\|_{H^{s}(0, T, V)}^{2}=\sum_{i=0}^{s} \int_{0}^{T}\left\|v^{(i)}(t)\right\|_{V}^{2} d t<\infty
$$

We use the notations $\mathscr{C}^{0}([0, T], V)=\mathscr{C}([0, T], V)$ and $H^{0}(0, T, V)=L^{2}(0, T, V)$. The space of essentially bounded Bochner measurable functions $v:(0, T) \rightarrow V$ is denoted by $L^{\infty}(0, T, V)$. In this space we consider the following norm

$$
\|v\|_{L^{\infty}(0, T, V)}=\underset{(0, T)}{\operatorname{ess} \sup \|v(t)\|_{V}} .
$$

We also consider the following space

$$
\mathscr{W}(0, T)=\left\{g \in L^{2}\left(0, T, H_{0}^{1}(0,1)\right): g^{\prime} \in L^{2}\left(0, T, H^{-1}(0,1)\right)\right\},
$$

where $H^{-1}(0,1)$ denotes the dual space of $H^{1}(0,1)$.

Thus we replace the IBVP (1)-(4) by the following variational problem (VP): find $c \in \mathscr{W}(0, T)$ such that

$$
\left\langle\frac{d c}{d t}(t), w\right\rangle+\left(a(c(t)) \frac{\partial c}{\partial x}(t), \frac{d w}{d x}\right)=-\int_{0}^{t} k_{e r}(t-s)\left(d(c(s)) \frac{\partial c}{\partial x}(s), \frac{d w}{d x}\right) d s+(f(t), w),
$$

a. e. in $(0, T), \forall w \in H_{0}^{1}(0,1)$,

where

$$
c(0)=c_{0},
$$

where $<., .>$ denotes the duality pairing between $H^{-1}(0,1)$ and $H^{1}(0,1)$.

The existence and uniqueness of the variational problem is established in [26] for general kernels $k_{e r}$ using the contraction mapping principle under appropriate conditions on $a$ and $d$, namely, the boundeness of $a, a^{\prime}, d$ and $d^{\prime}$ and the existence of a lower positive bound for $a^{\prime}$. For the kernel $k_{e r}$ it is enough to assume $k_{e r} \in L^{1}(0, T)$. It should be pointed out that existence and uniqueness of a solution of problems of type (8) have been studied in the literature using different techniques under smoother assumptions on the kernel $k_{e r}$ (see [26]). Weaker conditions on the coefficients are considered in [30] where coefficients were allowed to grow uniformly in time.

Let $h=\left(h_{1}, \ldots, h_{N}\right)$, with $h_{i}>0$, for $i=1, \ldots, N$, be such that $\sum_{i=1}^{N} h_{i}=1$. We define in $I=[0,1]$ the nonuniform grid

$$
I_{h}=\left\{x_{i}, i=0, \ldots, N, x_{i}=x_{i-1}+h_{i}, i=1, \ldots, N, x_{0}=0\right\}
$$


and we use the notations $I_{h}^{\prime}=I_{h}-\{0,1\}$ and $\partial I_{h}=\{0,1\}$.

By $\mathbb{W}_{h}$ we denote the space of grid functions defined in $I_{h}$ and by $P_{h}$ the piecewise linear interpolation operator defined in $\mathbb{W}_{h}$. By $\mathbb{W}_{h, 0}$ we denote the subspace of $\mathbb{W}_{h}$ of the grid functions null on $\partial I_{h}$. The piecewise linear approximation $\hat{c}_{h}(t)=P_{h} c_{h}(t)$ for the concentration $c(t)$ is a solution of the following equation

$$
\begin{aligned}
& \left(\frac{d \hat{c}_{h}}{d t}(t), P_{h} w_{h}\right)+\left(a\left(\hat{c}_{h}(t)\right) \frac{\partial \hat{c}_{h}}{\partial x}(t), \frac{d}{d x} P_{h} w_{h}\right) \\
& \quad=-\int_{0}^{t} k_{e r}(t-s)\left(d\left(\hat{c}_{h}(s)\right) \frac{\partial \hat{c}_{h}}{\partial x}(s), \frac{d}{d x} P_{h} w_{h}\right) d s+\left(f(t), P_{h} w_{h}\right), \forall w_{h} \in \mathbb{W}_{h, 0}
\end{aligned}
$$

with

$$
\hat{c}_{h}(0)=P_{h} R_{h} c_{0}
$$

where $R_{h}$ denotes the restriction operator $R_{h}: \mathscr{C}([0,1]) \rightarrow \mathbb{W}_{h}, R_{h} \phi(x)=\phi(x), x \in I_{h}, \phi \in \mathscr{C}([0,1])$.

Let $h_{i+\frac{1}{2}}=\frac{1}{2}\left(h_{i}+h_{i+1}\right), i=1, \ldots, N-1, x_{i \pm \frac{1}{2}}=\frac{1}{2}\left(x_{i \pm 1}+x_{i}\right)$. To define the semi-discrete approximation we introduce the following definitions:

$$
\left\{\begin{array}{l}
g_{h}\left(x_{i}\right)=\frac{1}{h_{i+\frac{1}{2}}} \int_{x_{i}-\frac{1}{2}}^{x_{i}+\frac{1}{2}} g(x) d x, i=1, \ldots, N-1, \\
g_{h}\left(x_{0}\right)=\frac{2}{h_{1}} \int_{0}^{x_{1}} g(x) d x \\
g_{h}\left(x_{N}\right)=\frac{2}{h_{N}} \int_{x_{N-\frac{1}{2}}}^{1} g(x) d x
\end{array}\right.
$$

and

$$
\begin{aligned}
& M_{h} v_{h}\left(x_{i}\right)=\frac{1}{2}\left(v_{h}\left(x_{i-1}\right)+v_{h}\left(x_{i}\right)\right), i=1, \ldots, N, \\
& M_{h} v_{h}\left(x_{0}\right)=0, v_{h} \in \mathbb{W}_{h, 0} .
\end{aligned}
$$

In $\mathbb{W}_{h, 0}$ we consider the discrete inner product

$$
\left(v_{h}, w_{h}\right)_{h}=\sum_{i=1}^{N-1} h_{i+\frac{1}{2}} v_{h}\left(x_{i}\right) w_{h}\left(x_{i}\right), v_{h}, w_{h} \in \mathbb{W}_{h, 0},
$$

and by $\|\cdot\|_{h}$ we denote the norm induced by the previous discrete inner product. In what follows we use the notations

$$
\left(v_{h}, w_{h}\right)_{h,+}=\sum_{i=1}^{N} h_{i} v_{h}\left(x_{i}\right) w_{h}\left(x_{i}\right), v_{h}, w_{h} \in \mathbb{W}_{h},
$$

and

$$
\left\|v_{h}\right\|_{h,+}=\left(v_{h}, v_{h}\right)_{h,+}^{1 / 2} .
$$

In the space $\mathbb{W}_{h}$ we introduce the norm $\|\cdot\|_{1, h}$ defined by

$$
\left\|u_{h}\right\|_{1, h}^{2}=\left\|v_{h}\right\|_{h}^{2}+\left\|D_{-x} v_{h}\right\|_{h,+}^{2},
$$

where $D_{-x}$ represent the usual backward finite difference operator. 
The semi-discrete approximation for the solution of the variational problem (10) and (11) is computed using the following differential problem: find $c_{h}:[0, T] \rightarrow \mathbb{W}_{h, 0}$ such that

$$
\begin{aligned}
& \left(\frac{d c_{h}}{d t}(t), w_{h}\right)_{h}+\left(a\left(M_{h} c_{h}(t)\right) D_{-x} c_{h}(t), D_{-x} w_{h}\right)_{h,+} \\
& \quad=-\int_{0}^{t} k_{e r}(t-s)\left(d\left(M_{h} c_{h}(s)\right) D_{-x} c_{h}(s), D_{-x} w_{h}\right)_{h,+} d s+\left(f_{h}(t), w_{h}\right)_{h},
\end{aligned}
$$

for all $w_{h} \in \mathbb{W}_{h, 0}$, and

$$
c_{h}(0)=R_{h} c_{0},
$$

where $f_{h}$ is defined by (12) with $g$ replaced by $f(t)$. This solution is absolutely continuous in time and if $\mathrm{f}$ is continuous then $c_{h} \in \mathscr{C}^{1}\left([0, T], W_{h}\right)$. The time derivative then exists in the classical sense for almost all $t$.

It is easy to show that $c_{h}$ is solution of the initial value problem (15), (16) if and only if $c_{h}$ satisfies

$$
\begin{aligned}
& \frac{d c_{h}}{d t}(t)-D_{x}^{*}\left(a\left(M_{h} c_{h}(t)\right) D_{-x} c_{h}(t)\right)=\int_{0}^{t} k_{e r}(t-s) D_{x}^{*}\left(d\left(M_{h} c_{h}(s)\right) D_{-x} c_{h}(s)\right) d s+f_{h}(t) \\
& \text { in } I_{h}^{\prime}, \\
& c_{h}(t)=0 \text { on } \partial I_{h},
\end{aligned}
$$

and (16).

In (17) $D_{x}^{*}$ denotes the following finite difference operator

$$
D_{x}^{*} v_{h}\left(x_{i}\right)=\frac{v_{h}\left(x_{i+1}\right)-v_{h}\left(x_{i}\right)}{h_{i+\frac{1}{2}}}, i=1, \ldots, N-1, v_{h} \in \mathbb{W}_{h}
$$

The local existence and uniqueness of the solution of the initial value problem (15), (16) or equivalently (17), (16), can be stated using the results presented, for instance, in [3] or [31]. If $a^{\prime}, d^{\prime}$ are continuous in a ball centered in $c_{h}(0)$ and $k_{e r}$ is bounded in a certain bounded time interval, then it can be shown the local existence and uniqueness for the solution of (16), (17) (see [3]).

\section{Error analysis}

Let $\Lambda$ be a sequence of vectors $h=\left(h_{1}, \ldots, h_{N}\right), h_{i}>0, i=1, \ldots, N, \sum_{i=1}^{N} h_{i}=1$, and $h_{\max }=\max _{i=1, \ldots, N} h_{i} \rightarrow 0$. For $h \in \Lambda$, let $e_{h}(t)=R_{h} c(t)-c_{h}(t)$ be the semi-discretization error induced by (15) and (16) or equivalently (17) and (16). Wheeler introduced in [37] an approach that is based on the following split of $e_{h}(t)$

$$
e_{h}(t)=\rho_{h}(t)+\theta(t),
$$

where $\rho_{h}(t)=R_{h} c(t)-\tilde{c}_{h}(t), \theta(t)=\tilde{c}_{h}(t)-c_{h}(t)$ being $\tilde{c}_{h}(t)$ the solution of an elliptic problem that depends on $t$. In [5] this approach was followed for a linear version of (1) and was proved $\left\|e_{h}(t)\right\|_{h}=O\left(h_{\max }^{2}\right)$ and $\left\|D_{-x} e_{h}(t)\right\|_{h,+}=O\left(h_{\max }^{2}\right)$ under the following smoothness assumption: $c \in$ $H^{1}\left(0, T, H^{3}(0,1)\right) \cap L^{2}\left(0, T, H^{3}(0,1) \cap H_{0}^{1}(0,1)\right)$.

The approach that we follow here was introduced in [24] to study finite difference schemes for the linear version of (17). This approach allows the weakening of the smoothness conditions usually required 
when Wheeler's technique is used, namely, the replacement of $c \in H^{1}\left(0, T, H^{3}(0,1)\right) \cap L^{2}\left(0, T, H^{3}(0,1) \cap\right.$ $\left.H_{0}^{1}(0,1)\right)$ by

$$
c \in H^{1}\left(0, T, H^{2}(0,1)\right) \cap L^{2}\left(0, T, H^{3}(0,1) \cap H_{0}^{1}(0,1)\right) .
$$

In the convergence analysis we require some smoothness to the solution $c$ of $(\mathbf{V P})$. We suppose that $c$ verifies (18). We also use the continuous embedding of $H^{1}\left(0, T, H^{2}(0,1)\right)$ into $L^{\infty}\left(0, T, W^{1, \infty}(0,1)\right)$ The discrete Poincaré-Friedrich's inequality

$$
\left\|v_{h}\right\|_{h}^{2} \leq\left\|D_{-x} v_{h}\right\|_{h,+}^{2}, v_{h} \in \mathbb{W}_{h, 0},
$$

will be used in the proof of Theorem 1 . By $\mathscr{C}_{B}^{1}(\mathbb{R})$ we represent the space of bounded continuous real functions with bounded first order derivative.

In the convergence analysis the smoothness of the kernel function $k_{e r}$ has a central role. In fact depending on such smoothness we get error estimates that hold for different classes of problems. Based on this fact and in order to see the influence of the regularity of $k_{e r}$ in the error estimates we separate the error analysis into two cases: non singular and weakly singular kernels.

\subsection{Square integrable kernels}

In this section we assume that $k_{e r} \in L^{2}(0, T)$. Lower smoothness will be imposed in the next section. This assumption can be easily verified in several applications. For instance the mathematical models for drug delivery from polymeric matrices that present a constant relaxation time are described by integrodifferential equations of type (1) where the kernels $k_{e r}$ are of type $k_{e r}(t)=\frac{d}{\tau} e^{-\frac{t}{\tau}}$ (see [27]). Moreover, equation (1) is also used to model diffusion in viscoelastic materials where the relation between stress and strain is described by a Maxwell fluid model, a three parameter solid (Voigt-Kelvin) model or MaxwellWiechert generalized model (see [7]).

Theorem 1. Let c be a solution of (VP), such that $c$ satisfies (18), and let $c_{h}$ be the approximation defined by (15). If $a, d \in \mathscr{C}_{B}^{1}(\mathbb{R}), 0<a_{0} \leq a$, and $k_{e r} \in L^{2}(0, T)$, then there exist positive constants $C_{1}$ and $C_{2}$ depending on the coefficient functions $a, d$ and on the kernel $k_{e r}$ such that

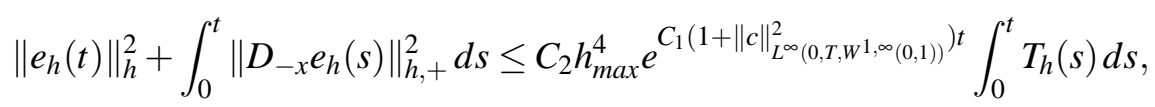

where

$$
\begin{aligned}
T_{h}(t) & =\left\|\frac{d c}{d t}(t)\right\|_{H^{2}(0,1)}^{2}+\left(1+\|c(t)\|_{W^{1, \infty}(0,1)}^{2}\right)\|c(t)\|_{H^{3}(0,1)}^{2} \\
& +\left(1+\|c\|_{L^{\infty}\left(0, T, W^{1, \infty}(0,1)\right)}^{2}\right) \int_{0}^{t}\|c(s)\|_{H^{3}(0,1)}^{2} d s
\end{aligned}
$$

Proof. From (15), it follows that $e_{h}(t)$ satisfies

$$
\begin{aligned}
\left(\frac{d e_{h}}{d t}(t), w_{h}\right)_{h}= & \left(R_{h} \frac{d c}{d t}(t), w_{h}\right)_{h}+\left(a\left(M_{h} c_{h}(t)\right) D_{-x} c_{h}(t), D_{-x} w_{h}\right)_{h,+} \\
& +\int_{0}^{t} k_{e r}(t-s)\left(d\left(M_{h} c_{h}(s)\right) D_{-x} c_{h}(s), D_{-x} w_{h}\right)_{h,+} d s \\
& -\left(f_{h}(t), w_{h}\right)_{h}, w_{h} \in \mathbb{W}_{h, 0} .
\end{aligned}
$$


We fix in (22) $w_{h}=e_{h}(t)$. We have

$$
\begin{aligned}
\left(f_{h}(t), e_{h}(t)\right)_{h}= & \left(\left(\frac{d c}{d t}\right)_{h}(t), e_{h}(t)\right)_{h}-\left(\left(\frac{\partial}{\partial x}\left(a(c(t)) \frac{\partial c}{\partial x}(t)\right)\right)_{h}, e_{h}(t)\right)_{h} \\
& -\left(\int_{0}^{t} k_{e r}(t-s)\left(\frac{\partial}{\partial x}\left(d(c(s)) \frac{\partial c}{\partial x}(s)\right)\right)_{h} d s, e_{h}(t)\right)_{h}
\end{aligned}
$$

where $\left(\frac{d c}{d t}\right)_{h}(t),\left(\frac{\partial}{\partial x}\left(a(c(t)) \frac{\partial c}{\partial x}(t)\right)\right)_{h}$ and $\left(\frac{\partial}{\partial x}\left(d(c(s)) \frac{\partial c}{\partial x}(s)\right)\right)_{h}$ are defined by (12) with $g$ replaced by $\frac{d c}{d t}(t), \frac{\partial}{\partial x}\left(a(c(t)) \frac{\partial c}{\partial x}(t)\right)$ and $\frac{\partial}{\partial x}\left(d(c(s)) \frac{\partial c}{\partial x}(s)\right)$, respectively.

Using integration in the third and fourth terms of the right hand side of (23), followed by summation by parts, it is easy to show that

$$
\left(\left(\frac{\partial}{\partial x}\left(a(c(t)) \frac{\partial c}{\partial x}(t)\right)\right)_{h}, e_{h}(t)\right)_{h}=-\left(a\left(\hat{M}_{h} c(t)\right) \hat{M}_{h} \frac{\partial c}{\partial x}(t), D_{-x} e_{h}(t)\right)_{h,+}
$$

and

$$
\left.\left(\frac{\partial}{\partial x}\left(d(c(s)) \frac{\partial c}{\partial x}(s)\right)\right)_{h}, e_{h}(t)\right)_{h}=-\left(d\left(\hat{M}_{h} c(s)\right) \hat{M}_{h} \frac{\partial c}{\partial x}(s), D_{-x} e_{h}(t)\right)_{h,+}
$$

where $\hat{M}_{h} g\left(x_{i}\right)=g\left(x_{i-\frac{1}{2}}\right), i=1, \ldots, N$.

From (22) with $w_{h}=e_{h}(t),(23)-(25)$ we deduce

$$
\frac{1}{2} \frac{d}{d t}\left\|e_{h}(t)\right\|_{h}^{2}=T_{a}(t)+T_{\text {int }}(t)+\sum_{p=1}^{3} Z_{p}(t),
$$

where

$$
\begin{gathered}
T_{a}(t)=\left(a\left(M_{h} c_{h}(t)\right) D_{-x} c_{h}(t), D_{-x} e_{h}(t)\right)_{h,+}-\left(a\left(M_{h} c(t)\right) D_{-x} R_{h} c(t), D_{-x} e_{h}(t)\right)_{h,+}, \\
T_{\text {int }}(t)=\int_{0}^{t} k_{e r}(t-s)\left(d\left(M_{h} c_{h}(s)\right) D_{-x} c_{h}(s)-d\left(M_{h} c(s)\right) D_{-x} R_{h} c(s), D_{-x} e_{h}(t)\right)_{h,+} d s \\
Z_{1}(t)=\left(R_{h} \frac{d c}{d t}(t)-\left(\frac{d c}{d t}\right)_{h}(t), e_{h}(t)\right)_{h}, \\
Z_{2}(t)=\left(a\left(M_{h} c(t)\right) D_{-x} R_{h} c(t)-a\left(\hat{M}_{h} c(t)\right) \hat{M}_{h} \frac{\partial c}{\partial x}(t), D_{-x} e_{h}(t)\right)_{h,+},
\end{gathered}
$$

and

$$
Z_{3}(t)=\int_{0}^{t} k_{e r}(t-s)\left(d\left(M_{h} c(s)\right) D_{-x} R_{h} c(s)-d\left(\hat{M}_{h} c(t)\right) \hat{M}_{h} \frac{\partial c}{\partial x}(s), D_{-x} e_{h}(t)\right)_{h,+} d s .
$$

We estimate separately the previous terms.

1. Estimate for $T_{a}(t)$ :

We have

$$
\begin{aligned}
T_{a}(t) & =\left(a\left(M_{h} c_{h}(t)\right) D_{-x} e_{h}(t), D_{-x} e_{h}(t)\right)_{h,+} \\
& +\left(\left(a\left(M_{h} c_{h}(t)\right)-a\left(M_{h} c(t)\right)\right) D_{-x} R_{h} c(t), D_{-x} e_{h}(t)\right)_{h,+}
\end{aligned}
$$

consequently, as $a \geq a_{0}>0$, we obtain 


$$
T_{a}(t) \leq-a_{0}\left\|D_{-x} e_{h}(t)\right\|_{h,+}^{2}+\frac{\left(a_{b}^{\prime}\right)^{2}}{4 \varepsilon_{0}^{2}}\left\|D_{-x} R_{h} c\right\|_{h,+}^{2}\left\|e_{h}(t)\right\|_{h}^{2}+\varepsilon_{0}^{2}\left\|D_{-x} e_{h}(t)\right\|_{h,+}^{2},
$$

where $\left|a^{\prime}\right| \leq a_{b}^{\prime}$ in $\mathbb{R}$ and $\varepsilon_{0} \neq 0$ is an arbitrary constant.

From (27) we conclude

$$
T_{a}(t) \leq\left(-a_{0}+\varepsilon_{0}^{2}\right)\left\|D_{-x} e_{h}(t)\right\|_{h,+}^{2}+\frac{\left(a_{b}^{\prime}\right)^{2}}{4 \varepsilon_{0}^{2}}\|c(t)\|_{W^{1, \infty}(0,1)}\left\|e_{h}(t)\right\|_{h}^{2} .
$$

2. Estimate for $T_{\text {int }}(t)$ :

As $d \in \mathscr{C}_{B}^{1}(\mathbb{R})$, following the procedure used to deduce (28), it can be shown that

$$
\begin{aligned}
\left|T_{\text {int }}(t)\right| \leq & d_{b} \int_{0}^{t}\left|k_{e r}(t-s)\right|\left\|D_{-x} e_{h}(s)\right\|_{h,+} d s\left\|D_{-x} e_{h}(t)\right\|_{h,+} \\
& +d_{b}^{\prime} \int_{0}^{t}\left|k_{e r}(t-s)\right|\left\|D_{-x} R_{h} c(s)\right\|_{h,+}\left\|e_{h}(s)\right\|_{h} d s\left\|D_{-x} e_{h}(t)\right\|_{h,+}
\end{aligned}
$$

and then, using the discrete Poincaré-Friedrichs inequality, we establish

$$
\begin{aligned}
\left|T_{\text {int }}(t)\right| \leq & \frac{1}{4 \varepsilon_{1}^{2}} k\left(d_{b}^{2}+\left(d_{b}^{\prime}\right)^{2}\|c\|_{L^{\infty}\left(0, T, W^{1, \infty}(0,1)\right)}^{2}\right) \int_{0}^{t}\left\|D_{-x} e_{h}(s)\right\|_{h,+}^{2} d s \\
& +2 \varepsilon_{1}^{2}\left\|D_{-x} e_{h}(t)\right\|_{h,+}^{2}
\end{aligned}
$$

where $k=\left\|k_{e r}\right\|_{L^{2}(0, T)}^{2},\left|d^{\prime}\right| \leq d_{b}^{\prime}$ in $\mathbb{R}$ and $\varepsilon_{1} \neq 0$ is an arbitrary constant.

3. Estimate for $Z_{1}(t)$ :

It can be shown that for $Z_{1}$ holds

$$
\left|Z_{1}(t)\right| \leq C_{Z_{1}} h_{\max }^{2}\left\|\frac{d c}{d t}(t)\right\|_{H^{2}(0,1)}\left\|D_{-x} e_{h}(t)\right\|_{h,+}
$$

where $C_{Z_{1}}$ is a positive constant (see [4]). Consequently we have

$$
\left|Z_{1}(t)\right| \leq \frac{1}{4 \varepsilon_{2}^{2}} C_{Z_{1}}^{2} h_{\max }^{4}\left\|\frac{d c}{d t}(t)\right\|_{H^{2}(0,1)}^{2}+\varepsilon_{2}^{2}\left\|D_{-x} e_{h}(t)\right\|_{h,+}^{2}
$$

where $\varepsilon_{2} \neq 0$ is an arbitrary constant.

4. Estimate for $Z_{2}(t)$ :

For $Z_{2}$ holds the representation

$$
Z_{2}(t)=Z_{2,1}(t)+Z_{2,2}(t)
$$

with

$$
Z_{2,1}(t)=\left(a\left(\hat{M}_{h} c(t)\right)\left(D_{-x} R_{h} c(t)-\hat{M}_{h} \frac{\partial c}{\partial x}(t)\right), D_{-x} e_{h}(t)\right)_{h,+}
$$


and

$$
Z_{2,2}(t)=\left(\left(a\left(M_{h} c(t)\right)-a\left(\hat{M}_{h} c(t)\right)\right) D_{-x} R_{h} c(t), D_{-x} e_{h}(t)\right)_{h,+} .
$$

To estimate $Z_{2,1}(t)$ we remak that

$$
Z_{2,1}(t)=\left(a\left(\hat{M}_{h} c(t)\right) \lambda(g), D_{-x} e_{h}(t)\right)_{h,+},
$$

with $g(\xi)=c\left(x_{i-1}+\xi h_{i}, t\right)$ and

$$
\lambda(g)=\frac{1}{h_{i}}\left(g(1)-g(0)-g^{\prime}\left(\frac{1}{2}\right)\right) .
$$

Applying the Bramble-Hilbert lemma ([6]) to estimate $\lambda(g)$ we obtain

$$
|\lambda(g)| \leq C_{Z_{2,1}} h_{i}\left|\frac{\partial^{3} c}{\partial x^{3}}(t)\right|_{L^{1}\left(x_{i-1}, x_{i}\right)},
$$

where $C_{Z_{2,1}}$ is a positive constant. The last estimate leads to

$$
\left|Z_{2,1}(t)\right| \leq a_{b} C_{Z_{2,1}} h_{\max }^{2}|c(t)|_{H^{3}(0,1)}\left\|D_{-x} e_{h}(t)\right\|_{h,+}
$$

which implies

$$
\left|Z_{2,1}(t)\right| \leq \frac{a_{b}^{2} C_{Z_{2,1}}^{2}}{4 \varepsilon_{3}^{2}} h_{\max }^{4}|c(t)|_{H^{3}(0,1)}^{2}+\varepsilon_{3}^{2}\left\|D_{-x} e_{h}(t)\right\|_{h,+}^{2},
$$

where $a \leq a_{b}$ in $\mathbb{R}$ and $\varepsilon_{3} \neq 0$ is an arbitrary constant.

To estimate $Z_{2,2}(t)$ we consider

$$
\lambda(g)=\frac{1}{2}(g(1)+g(0))-g\left(\frac{1}{2}\right)
$$

with $g(\xi)=c\left(x_{i-1}+\xi h_{i}, t\right)$. Applying the Bramble-Hilbert lemma to estimate $\lambda(g)$ we obtain

$$
|\lambda(g)| \leq C_{Z_{2,2}} h_{i}\left|\frac{\partial^{2} c}{\partial x^{2}}(t)\right|_{L^{1}\left(x_{i-1}, x_{i}\right)},
$$

where $C_{Z_{2,2}}$ is a positive constant. Then

$$
\left|Z_{2,2}(t)\right| \leq a_{b}^{\prime} C_{Z_{2,2}} h_{\max }^{2}\left\|D_{-x} R_{h} c(t)\right\|_{h,+}|c(t)|_{H^{2}(0,1)}\left\|D_{-x} e_{h}(t)\right\|_{h,+},
$$

which implies

$$
\left|Z_{2,2}(t)\right| \leq \frac{\left(a_{b}^{\prime}\right)^{2} C_{Z_{2,2}}^{2}}{4 \varepsilon_{4}^{2}} h_{\text {max }}^{4}\|c(t)\|_{W^{1, \infty}(0,1)}^{2}|c(t)|_{H^{2}(0,1)}^{2}+\varepsilon_{4}^{2}\left\|D_{-x} e_{h}(t)\right\|_{h,+}^{2},
$$

where $\left|a^{\prime}\right| \leq a_{b}^{\prime}$ in $\mathbb{R}$ and $\varepsilon_{4} \neq 0$ is an arbitrary constant.

Then from (33) and (35), there exists a positive constant $C_{Z_{2}}$ such that

$$
\begin{aligned}
\left|Z_{2}(t)\right| \leq & C_{Z_{2}} h_{\max }^{4}\left(\frac{a_{b}^{2}}{4 \varepsilon_{3}^{2}}\|c(t)\|_{H^{3}(0,1)}^{2}+\frac{\left(a_{b}^{\prime}\right)^{2}}{4 \varepsilon_{4}^{2}}\|c(t)\|_{W^{1, \infty}(0,1)}^{2}\|c(t)\|_{H^{2}(0,1)}^{2}\right) \\
& +\left(\varepsilon_{3}^{2}+\varepsilon_{4}^{2}\right)\left\|D_{-x} e_{h}^{n}\right\|_{h,+}^{2}
\end{aligned}
$$




\section{Estimate for $Z_{3}(t)$ :}

Following the steps used to estimate $Z_{2}(t)$ it can be shown that

$$
\begin{aligned}
\left|Z_{3}(t)\right| & \leq \int_{0}^{t}\left|k_{e r}(t-s)\right| d_{b} C_{Z_{3,1}} h_{\max }^{2}|c(s)|_{H^{3}(0,1)} d s\left\|D_{-x} e_{h}(t)\right\|_{h,+} \\
& +\int_{0}^{t}\left|k_{e r}(t-s)\right| d_{b}^{\prime} C_{Z_{3,2}} h_{\max }^{2}|c(s)|_{H^{2}(0,1)}\left\|D_{-x} R_{h} c(s)\right\|_{h,+} d s\left\|D_{-x} e_{h}(t)\right\|_{h,+}
\end{aligned}
$$

where $|d| \leq d_{b}$ and $\left|d^{\prime}\right| \leq d_{b}^{\prime}$ in $\mathbb{R}$.

As $k_{e r} \in L^{2}(0, T)$, from (37) we get

$$
\begin{aligned}
\left|Z_{3}(t)\right| & \leq h_{\max }^{4} \frac{1}{4 \varepsilon_{5}^{2}} k C_{Z_{3}}\left(d_{b}^{2}+\left(d_{b}^{\prime}\right)^{2}\|c\|_{L^{\infty}\left(0, T, W^{1, \infty}(0,1)\right)}^{2}\right) \int_{0}^{t}\|c(s)\|_{H^{3}(0,1)}^{2} d s \\
& +2 \varepsilon_{5}^{2}\left\|D_{-x} e_{h}(t)\right\|_{h,+}^{2},
\end{aligned}
$$

where $\varepsilon_{5} \neq 0$ is an arbitrary constant.

Considering in (28)-(38) $\varepsilon_{i}=\varepsilon, i=0, \ldots, 5$, and taking in (26) these upper bounds we obtain

$$
\begin{aligned}
\frac{d}{d t}\left\|e_{h}(t)\right\|_{h}^{2} & +2\left(a_{0}-8 \varepsilon^{2}\right)\left\|D_{-x} e_{h}(t)\right\|_{h,+}^{2} \leq \frac{\left(a_{b}^{\prime}\right)^{2}}{2 \varepsilon^{2}}\|c(t)\|_{W^{1, \infty}(0,1)}^{2}\left\|e_{h}(t)\right\|_{h}^{2} \\
& +\frac{1}{2 \varepsilon^{2}} k\left(d_{b}^{2}+\left(d_{b}^{\prime}\right)^{2}\|c\|_{L^{\infty}\left(0, T, W^{1, \infty}(0,1)\right)}^{2}\right) \int_{0}^{t}\left\|D_{-x} e_{h}(t)\right\|_{h,+}^{2} d s \\
& +h_{\max }^{4} \frac{1}{2 \varepsilon^{2}} C_{T} T_{h}(t),
\end{aligned}
$$

where $T_{h}(t)$ is defined by (21) and $C_{T}$ is given by

$$
C_{T}=\max \left\{C_{Z_{1}}^{2}, a_{b}^{2} C_{Z_{2}},\left(a_{b}^{\prime}\right)^{2} C_{Z_{2}}, k d_{b}^{2} C_{Z_{3}}, k\left(d_{b}^{\prime}\right)^{2} C_{Z_{3}}\right\} .
$$

Inequality (39) leads to

$$
\begin{aligned}
\left\|e_{h}(t)\right\|_{h}^{2} & +2\left(a_{0}-8 \varepsilon^{2}\right) \int_{0}^{t}\left\|D_{-x} e_{h}(s)\right\|_{h,+}^{2} d s \leq \frac{\left(a_{b}^{\prime}\right)^{2}}{2 \varepsilon^{2}} \int_{0}^{t}\|c(s)\|_{W^{1, \infty}(0,1)}^{2}\left\|e_{h}(s)\right\|_{h}^{2} d s \\
& +\frac{1}{2 \varepsilon^{2}} k\left(d_{b}^{2}+\left(d_{b}^{\prime}\right)^{2}\|c\|_{L^{\infty}\left(0, T, W^{1, \infty}(0,1)\right)}^{2}\right) \int_{0}^{t} \int_{0}^{s}\left\|D_{-x} e_{h}(\mu)\right\|_{h,+}^{2} d \mu d s \\
& +h_{\max }^{4} \frac{1}{2 \varepsilon^{2}} C_{T} \int_{0}^{t} T_{h}(s) d s
\end{aligned}
$$

that implies

$$
\begin{aligned}
& \left\|e_{h}(t)\right\|_{h}^{2}+\int_{0}^{t}\left\|D_{-x} e_{h}(s)\right\|_{h,+}^{2} d s \leq \frac{\left(a_{b}^{\prime}\right)^{2}\|c\|_{L^{\infty}\left(0, T, W^{1, \infty}(0,1)\right)}^{2}}{2 \varepsilon^{2} \min \left\{1,2\left(a_{0}-8 \varepsilon^{2}\right)\right\}} \int_{0}^{t}\left\|e_{h}(s)\right\|_{h}^{2} d s \\
& +\frac{1}{2 \varepsilon^{2} \min \left\{1,2\left(a_{0}-8 \varepsilon^{2}\right)\right\}} k\left(d_{b}^{2}+\left(d_{b}^{\prime}\right)^{2}\|c\|_{L^{\infty}\left(0, T, W^{1, \infty}(0,1)\right)}^{2}\right) \int_{0}^{t} \int_{0}^{s}\left\|D_{-x} e_{h}(\mu)\right\|_{h,+}^{2} d \mu d s \\
& +h_{\max }^{4} \frac{1}{2 \varepsilon^{2} \min \left\{1,2\left(a_{0}-8 \varepsilon^{2}\right)\right\}} C_{T} \int_{0}^{t} T_{h}(s) d s
\end{aligned}
$$


when $\varepsilon$ is fixed by

$$
a_{0}-8 \varepsilon^{2}>0
$$

From (41) we conclude that there exist positive constants $C_{1}$ and $C_{2}$ depending on the coefficients functions $a$ and $d$ and on the kernel function $k_{e r}$ such that

$$
\begin{aligned}
\left\|e_{h}(t)\right\|_{h}^{2} & +\int_{0}^{t}\left\|D_{-x} e_{h}(s)\right\|_{h,+}^{2} d s \leq \\
& C_{1}\left(1+\|c\|_{L^{\infty}\left(0, T, W^{1, \infty}(0,1)\right)}^{2}\right) \int_{0}^{t}\left(\left\|e_{h}(s)\right\|_{h}^{2}+\int_{0}^{s}\left\|D_{-x} e_{h}(\mu)\right\|_{h,+}^{2} d \mu\right) d s \\
& +C_{2} h_{\max }^{4} \int_{0}^{t} T_{h}(s) d s .
\end{aligned}
$$

Finally the application of the Gronwall lemma leads to (20).

In the upper bound (20), we have an exponential amplification factor $e^{\Theta t}$ where $\Theta=C_{1}(1+$ $\left.\|c\|_{L^{\infty}\left(0, T, W^{1, \infty}(0,1)\right)}^{2}\right)$. In certain situations this amplification factor can be reduced to the unity by considering more strict conditions on the coefficients.

\subsection{General weakly singular kernels}

In what follows we replace the smoothness assumption $k_{e r} \in L^{2}(0, T)$ by the following weaker condition $k_{e r} \in L^{1}(0, T)$. However, as will see, such a replacement implies a restriction in the class of problems that allow us to obtain the accuracy of the semi-discrete approximation $c_{h}(t)$ stated in Theorem 1.

In the proof of Theorem 1, the assumption $k_{e r} \in L^{2}(0, T)$ was used in the establishment of the upper bounds for $T_{\text {int }}(t)$ and and for $Z_{3}(t)$, respectively, (30) and (38). In what follows we get new estimates for these two terms assuming $k_{e r} \in L^{1}(0, T)$.

- Estimate for $T_{\text {int }}(t)$ :

From (29) we obtain

$$
\begin{aligned}
\left|T_{\text {int }}(t)\right| \leq & \frac{1}{4 \varepsilon_{1}^{2}} k\left(d_{b}^{2}+\left(d_{b}^{\prime}\right)^{2}\|c\|_{L^{\infty}\left(0, T, W^{1, \infty}(0,1)\right)}^{2}\right) \int_{0}^{t}\left|k_{e r}(t-s)\right|\left\|D_{-x} e_{h}(s)\right\|_{h,+}^{2} d s \\
& +2 \varepsilon_{1}^{2}\left\|D_{-x} e_{h}(t)\right\|_{h,+}^{2}
\end{aligned}
$$

where $k=\left\|k_{e r}\right\|_{L^{1}(0, T)}^{2}$.

- Estimate for $Z_{3}(t)$ :

It can be shown that for $Z_{3}(t)$ holds the following

$$
\begin{aligned}
\left|Z_{3}(t)\right| & \leq h_{\max }^{4} \frac{1}{4 \varepsilon_{5}^{2}} k C_{Z_{3}}\left(d_{b}^{2}+\left(d_{b}^{\prime}\right)^{2}\|c\|_{L^{\infty}\left(0, T, W^{1, \infty}(0,1)\right)}^{2}\right) \int_{0}^{t}\left|k_{e r}(t-s)\right|\|c(s)\|_{H^{3}(0,1)}^{2} d s \\
& +2 \varepsilon_{5}^{2}\left\|D_{-x} e_{h}(t)\right\|_{h,+}^{2},
\end{aligned}
$$

where $\varepsilon_{5} \neq 0$ is an arbitrary constant. 
Following the proof of Theorem 1, we obtain

$$
\begin{aligned}
\left\|e_{h}(t)\right\|_{h}^{2} & +2\left(a_{0}-8 \varepsilon^{2}\right) \int_{0}^{t}\left\|D_{-x} e_{h}(s)\right\|_{h,+}^{2} d s \leq \frac{\left(a_{b}^{\prime}\right)^{2}}{2 \varepsilon^{2}} \int_{0}^{t}\|c(s)\|_{W^{1, \infty}(0,1)}^{2}\left\|e_{h}(s)\right\|_{h}^{2} d s \\
& +\frac{1}{2 \varepsilon^{2}} k\left(d_{b}^{2}+\left(d_{b}^{\prime}\right)^{2}\|c\|_{L^{\infty}\left(0, T, W^{1, \infty}(0,1)\right)}^{2}\right) \int_{0}^{t} \int_{0}^{s} \mid k_{e r}(s-\mu)\left\|D_{-x} e_{h}(\mu)\right\|_{h,+}^{2} d \mu d s \\
& +h_{\max }^{4} \frac{1}{2 \varepsilon^{2}} C_{T} \int_{0}^{t} T_{h}(s) d s
\end{aligned}
$$

that replaces (40). In (46) $T_{h}(s)$ is defined now by

$$
\begin{aligned}
T_{h}(t) & =\left\|\frac{d c}{d t}(t)\right\|_{H^{2}(0,1)}^{2}+\left(1+\|c(t)\|_{W^{1, \infty}(0,1)}^{2}\right)\|c(t)\|_{H^{3}(0,1)}^{2} \\
& +\left(1+\|c\|_{L^{\infty}\left(0, T, W^{1, \infty}(0,1)\right)}^{2}\right) \int_{0}^{t}\left|k_{e r}(t-s)\right|\|c(s)\|_{H^{3}(0,1)}^{2} d s .
\end{aligned}
$$

As inequality (46) is equivalent to

$$
\begin{aligned}
\left\|e_{h}(t)\right\|_{h}^{2} & +2\left(a_{0}-8 \varepsilon^{2}\right) \int_{0}^{t}\left\|D_{-x} e_{h}(s)\right\|_{h,+}^{2} d s \leq \frac{\left(a_{b}^{\prime}\right)^{2}}{2 \varepsilon^{2}} \int_{0}^{t}\|c(s)\|_{W^{1, \infty}(0,1)}^{2}\left\|e_{h}(s)\right\|_{h}^{2} d s \\
& +\frac{1}{2 \varepsilon^{2}} k\left(d_{b}^{2}+\left(d_{b}^{\prime}\right)^{2}\|c\|_{L^{\infty}\left(0, T, W^{1, \infty}(0,1)\right)}^{2}\right) \int_{0}^{t} \int_{\mu}^{t} \mid k_{e r}(s-\mu)\left\|D_{-x} e_{h}(\mu)\right\|_{h,+}^{2} d s d \mu \\
& +h_{\max }^{4} \frac{1}{2 \varepsilon^{2}} C_{T} \int_{0}^{t} T_{h}(s) d s
\end{aligned}
$$

we get

$$
\begin{aligned}
\left\|e_{h}(t)\right\|_{h}^{2} & +2\left(a_{0}-8 \varepsilon^{2}\right) \int_{0}^{t}\left\|D_{-x} e_{h}(s)\right\|_{h,+}^{2} d s \leq h_{\max }^{4} \frac{1}{2 \varepsilon^{2}} C_{T} \int_{0}^{t} T_{h}(s) d s \\
& \left.+\frac{1}{2 \varepsilon^{2}}\left(k^{2} d_{b}^{2}+\left(k^{2}\left(d_{b}^{\prime}\right)^{2}\right)+\left(a_{b}^{\prime}\right)^{2}\right)\|c\|_{L^{\infty}\left(0, T, W^{1, \infty}(0,1)\right)}^{2}\right) \int_{0}^{t}\left\|D_{-x} e_{h}(\mu)\right\|_{h,+}^{2} d \mu .
\end{aligned}
$$

Under the following condition

$$
\left.2\left(a_{0}-8 \varepsilon^{2}\right)-\frac{1}{2 \varepsilon^{2}}\left(k^{2} d_{b}^{2}+\left(k^{2}\left(d_{b}^{\prime}\right)^{2}\right)+\left(a_{b}^{\prime}\right)^{2}\right)\|c\|_{L^{\infty}\left(0, T, W^{1, \infty}(0,1)\right)}^{2}\right)>0
$$

we conclude that

$$
\left\|e_{h}(t)\right\|_{h}^{2}+\int_{0}^{t}\left\|D_{-x} e_{h}(s)\right\|_{h,+}^{2} d s \leq C h_{\max }^{4} \int_{0}^{t} T_{h}(s) d s .
$$

for some positive constant $C$.

\section{An IMEX method}

\subsection{Non singular kernels}

To integrate in time an IMEX (implicit-explicit) method will be used. In $[0, T]$ we consider a time grid $J_{\Delta t}=\left\{t_{n}, n=0,1,2, \ldots, M\right\}$ with $t_{0}=0, t_{M}=T$ and $t_{n}-t_{n-1}=\Delta t$. We use the rectangular rule 
to approximate the integral in (1) and the backward finite-difference operator $D_{-t}$ to approximate the first partial derivative with respect to $t$. Then the fully discrete approximation for $c$ at $\left(x_{j}, t_{n}\right), c_{h}^{n}\left(x_{j}\right)$, is defined by the following set of equations

$$
\begin{aligned}
D_{-t} c_{h}^{n}\left(x_{j}\right)= & D_{x}^{*}\left(a\left(M_{h} c_{h}^{n-1}\left(x_{j}\right)\right) D_{-x} c_{h}^{n}\left(x_{j}\right)\right)+f\left(x_{j}, t_{n}\right) \\
& +\Delta t \sum_{\ell=0}^{n-1} k_{e r}\left(t_{n}-t_{\ell}\right) D_{x}^{*}\left(d\left(M_{h} c_{h}^{\ell}\left(x_{j}\right)\right) D_{-x} c_{h}^{\ell}\left(x_{j}\right)\right), \\
& j=1, \ldots, N-1,
\end{aligned}
$$

with boundary conditions

$$
c_{h}^{n}\left(x_{0}\right)=c_{h}^{n}\left(x_{N}\right)=0, \text { for } n=1, \ldots, M
$$

and the initial condition

$$
c_{h}^{0}\left(x_{j}\right)=R_{h} c_{0}\left(x_{j}\right), \text { for } j=1, \ldots, N-1,
$$

To compute the fully discrete solution at time level $t_{n}, c_{h}^{n}$, we need to solve a linear system $A_{h}\left(c_{h}^{n-1}\right) c_{h}^{n}=B$, where $A_{h}\left(c_{h}^{n-1}\right)$ is a tridiagonal matrix. Since the coefficient $a$ is positive, then $A_{h}\left(c_{h}^{n-1}\right)$ is strictly diagonal dominant and consequently $A_{h}\left(c_{H}^{n-1}\right)$ is a M-matrix.

We remark that the previous fully discrete space-time scheme can be written in the following equivalent form

$$
\begin{gathered}
\left(D_{-t} c_{h}^{n}, w_{h}\right)_{h}=-\left(a\left(M_{h} c_{h}^{n-1}\right) D_{-x} c_{h}^{n}, D_{-x} w_{h}\right)_{h,+}+\left(f_{h}\left(t_{n}\right), w_{h}\right)_{h} \\
-\Delta t \sum_{\ell=0}^{n-1} k_{e r}\left(t_{n}-t_{\ell}\right)\left(d\left(M_{h} c_{h}^{\ell}\right) D_{-x} c_{h}^{\ell}, D_{-x} w_{h}\right)_{h,+}, n=1, \ldots, M,
\end{gathered}
$$

for all $w_{h} \in \mathbb{W}_{h, 0}$, with the initial condition (52).

Let $c$ be a solution of (1), (2), (3) and let $e_{h}^{n}=R_{h} c\left(t_{n}\right)-c_{h}^{n}, n=0, \ldots, M$, be the global error. As the integral term was discretized using the rectangular grid, in order to obtain an estimate for $e_{h}^{n}$ we need to replace the assumption $k_{e r} \in L^{2}(0, T)$ by the following one

$$
k_{\text {er }} \in H^{1}(0, T) .
$$

Theorem 2. Let $c \in \mathscr{C}\left([0, T], H^{3}(0,1) \cap H_{0}^{1}(0,1)\right) \cap \mathscr{C}^{1}\left([0, T], H^{2}(0,1)\right)$ be the solution of $(\mathbf{V P})$ and let $c_{h}^{n}$ be its approximation defined by (50). If $a, d \in \mathscr{C}_{B}^{1}(\mathbb{R}), 0<a_{0} \leq a$, and $k_{\text {er }}$ satisfies (54), then there exists positive constant $C_{T}$ that does not depend on $h, \Delta t$ neither $c$, such that for the fully discrete error $e_{h}^{n}=R_{h} c\left(t_{n}\right)-c_{h}^{n}$ holds the following

$$
\begin{aligned}
\left\|e_{h}^{n}\right\|_{h}^{2} & +\Delta t \sum_{\ell=0}^{n}\left\|D_{-x} e_{h}^{\ell}\right\|_{h,+}^{2} \leq \exp \left(T \frac{\max \left\{\Phi+2 \varepsilon^{2}, \Psi\right\}}{\min \left\{1-\Delta t 2 \varepsilon^{2}, 2\left(a_{0}-12 \varepsilon^{2}\right)\right\}}\right) \\
& \frac{1}{\min \left\{1-\Delta t 2 \varepsilon^{2}, 2\left(a_{0}-12 \varepsilon^{2}\right)\right\}}\left(\left\|e_{h}^{0}\right\|_{h,+}^{2}+2\left(a_{0}-12 \varepsilon^{2}\right) \Delta t\left\|D_{-x} e_{h}^{0}\right\|_{h,+}^{2}+\Delta t \sum_{\ell=1}^{n} T_{h}^{\ell}\right),
\end{aligned}
$$

where $T_{h}^{\ell}$ is given by

$$
\begin{aligned}
T_{h}^{\ell}=C_{T} \frac{1}{2 \varepsilon^{2}} & h_{\max }^{4}\left(\|c\|_{\mathscr{C}^{1}\left([0, T], H^{2}(0,1)\right)}^{2}+\left(a_{b}^{2}+\left(a_{b}^{\prime}\right)^{2}\|c\|_{\mathscr{C}\left([0, T], W^{1, \infty}(0,1)\right)}^{2}\right)\|c\|_{\mathscr{C}\left([0, T], H^{3}(0,1)\right)}^{2}\right. \\
& +\left(d_{b}^{2}+\left(d_{b}^{\prime}\right)^{2}\|c\|_{\left.\mathscr{C}\left([0, T], W^{1, \infty}(0,1)\right)\right)}^{2}\|c\|_{L^{2}\left(0, T, H^{3}(0,1)\right)}^{2}\right) \\
& +\Delta t\left(\left\|R_{h} c\right\|_{H^{2}\left(t_{\ell-1}, t_{\ell}, \mathbb{W}_{h}\right)}^{2}+\left(a_{b}^{\prime}\right)^{2}\left\|R_{h} c\right\|_{H^{1}\left(t_{\ell-1}, t_{\ell}, \mathbb{W}_{h}\right)}^{2}\|c\|_{\mathscr{C}\left([0, T], W^{1, \infty}(0,1)\right)}^{2}\right) \\
& \left.+\Delta t^{2}\left(2 d_{b}^{2}\|c\|_{H^{1}\left(0, T, W^{1, \infty}(0,1)\right)}^{2}+\left(d_{b}^{\prime}\right)^{2}\|c\|_{\mathscr{C}\left([0, T], W^{1, \infty}(0,1)\right)}^{2}\left\|R_{h} c\right\|_{H^{1}\left(0, T, \mathbb{W}_{h}\right)}^{2}\right)\right)
\end{aligned}
$$


$\varepsilon$ is such that

$$
a_{0}-12 \varepsilon^{2}>0
$$

and $\Delta t$ is fixed by

$$
1-\Delta t 2 \varepsilon^{2}>0
$$

In (55), $\Phi$ and $\Psi$ are defined by

$$
\begin{gathered}
\Phi=\frac{\left(a_{b}^{\prime}\right)^{2}}{2 \varepsilon^{2}}\|c\|_{\mathscr{C}\left([0, T], W^{1, \infty}(0,1)\right)}^{2}, \\
\Psi=\frac{k}{2 \varepsilon^{2}}\left(d_{b}^{2}+\left(d_{b}^{\prime}\right)^{2}\|c\|_{\mathscr{C}\left([0, T], W^{1, \infty}(0,1)\right)}^{2}\right),
\end{gathered}
$$

respectively, and $k=T\left\|k_{e r}\right\|_{0, \infty}^{2}$.

Proof. Following the semi-discrete error analysis, it can be shown that

$$
\left(D_{-t} e_{h}^{n}, e_{h}^{n}\right)_{h}=T_{a}^{n}+T_{i n t, d}^{n}+\sum_{p=1}^{3} Z_{p}^{n}
$$

where $T_{a}^{n}, T_{i n t, d}^{n}, Z_{p}^{n}, p=1,2,3$, are given by

$$
\begin{gathered}
T_{a}^{n}=\left(a\left(M_{h} c_{h}^{n-1}\right) D_{-x} c_{h}^{n}, D_{-x} e_{h}^{n}\right)_{h,+}-\left(a\left(M_{h} c\left(t_{n-1}\right)\right) D_{-x} R_{h} c\left(t_{n}\right), D_{-x} e_{h}^{n}\right)_{h,+} \\
T_{i n t, d}^{n}=\Delta t \sum_{\ell=0}^{n-1} k_{e r}\left(t_{n}-t_{\ell}\right)\left(\left(d\left(M_{h} c_{h}^{\ell}\right) D_{-x} c_{h}^{\ell}, D_{-x} e_{h}^{n}\right)_{h,+}-\left(d\left(M_{h} c\left(t_{\ell}\right)\right) D_{-x} R_{h} c\left(t_{\ell}\right), D_{-x} e_{h}^{n}\right)_{h,+}\right) \\
Z_{1}^{n}=\left(D_{-t} R_{h} c\left(t_{n}\right)-\left(\frac{d c}{d t}\right)_{h}\left(t_{n}\right), e_{h}^{n}\right)_{h} \\
Z_{2}^{n}=\left(a\left(M_{h} c\left(t_{n-1}\right)\right) D_{-x} R_{h} c\left(t_{n}\right)-a\left(\hat{M}_{h} c\left(t_{n}\right)\right) \hat{M}_{h} \frac{\partial c}{\partial x}\left(t_{n}\right), D_{-x} e_{h}^{n}\right)_{h,+}
\end{gathered}
$$

and

$$
\begin{aligned}
Z_{3}^{n}=\Delta t & \sum_{\ell=0}^{n-1} k_{e r}\left(t_{n}-t_{\ell}\right)\left(d\left(M_{h} c\left(t_{\ell}\right)\right) D_{-x} R_{h} c\left(t_{\ell}\right), D_{-x} e_{h}^{n}\right)_{h,+} \\
& \quad-\int_{0}^{t_{n}} k_{e r}\left(t_{n}-s\right)\left(d\left(\hat{M}_{h} c(s)\right) \hat{M}_{h} \frac{\partial c}{\partial x}(s), D_{-x} e_{h}^{n}\right)_{h,+} d s .
\end{aligned}
$$

We estimate in what follows the introduced quantities:

- Estimate for $T_{a}^{n}$ : Following the proof of estimate (28) it can be shown that

$$
T_{a}^{n} \leq\left(-a_{0}+\varepsilon_{0}^{2}\right)\left\|D_{-x} e_{h}^{n}\right\|_{h,+}^{2}+\frac{\left(a_{b}^{\prime}\right)^{2}}{4 \varepsilon_{0}^{2}}\|c\|_{\mathscr{C}\left([0, T], W^{1, \infty}(0,1)\right)}^{2}\left\|e_{h}^{n-1}\right\|_{h}^{2}
$$

where $\varepsilon_{0} \neq 0$.

- Estimate for $T_{i n t, d}^{n}$ : Analogously to (44) we have

$$
\begin{aligned}
\left|T_{i n t, d}^{n}\right| \leq & \Delta t \frac{1}{4 \varepsilon_{1}^{2}} k\left(d_{b}^{2}+\left(d_{b}^{\prime}\right)^{2}\|c\|_{\mathscr{C}\left([0, T], W^{1, \infty}(0,1)\right)}^{2}\right) \sum_{\ell=0}^{n-1}\left\|D_{-x} e_{h}^{\ell}\right\|_{h,+}^{2} \\
& +2 \varepsilon_{1}^{2}\left\|D_{-x} e_{h}^{n}\right\|_{h,+}^{2}
\end{aligned}
$$

where $\varepsilon_{1} \neq 0$. 
- Estimate for $Z_{1}^{n}$ : As $Z_{1}^{n}$ admits the representation

$$
Z_{1}^{n}=\left(D_{-t} R_{h} c\left(t_{n}\right)-R_{h} \frac{\partial c}{\partial t}\left(t_{n}\right), e_{h}^{n}\right)_{h}+\left(R_{h} \frac{\partial c}{\partial t}\left(t_{n}\right)-\left(\frac{\partial c}{\partial t}\right)_{h}\left(t_{n}\right), e_{h}^{n}\right)_{h},
$$

we easily get

$$
\begin{aligned}
\left|Z_{1}^{n}\right| \leq & C_{Z_{1}}\left(\frac{1}{4 \varepsilon_{2}^{2}} \Delta t\left\|R_{h} c\right\|_{H^{2}\left(t_{n-1}, t_{n}, \mathbb{W}_{h}\right)}^{2}+\frac{1}{4 \varepsilon_{3}^{2}} h_{\max }^{4}\|c\|_{\mathscr{C}^{1}\left([0, T], H^{2}(0,1)\right)}^{2}\right)+\varepsilon_{2}^{2}\left\|e_{h}^{n}\right\|_{h}^{2} \\
& +\varepsilon_{3}^{2}\left\|D_{-x} e_{h}^{n}\right\|_{h,+}^{2},
\end{aligned}
$$

where $\varepsilon_{2}, \varepsilon_{3} \neq 0$.

- Estimate for $Z_{2}^{n}$ : We split $Z_{2}^{n}$ into $Z_{2}^{n}=Z_{2,1}^{n}+Z_{2,2}^{n}$ where

$$
Z_{2,1}^{n}=\left(a\left(M_{h} c\left(t_{n}\right)\right) D_{-x} R_{h} c\left(t_{n}\right)-a\left(\hat{M}_{h} c\left(t_{n}\right)\right) \hat{M}_{h} \frac{\partial c}{\partial x}\left(t_{n}\right), D_{-x} e_{h}^{n}\right)_{h,+}
$$

and

$$
Z_{2,2}^{n}=\left(\left(a\left(M_{h} c\left(t_{n-1}\right)\right)-a\left(M_{h} c\left(t_{n}\right)\right)\right) D_{-x} R_{h} c\left(t_{n}\right), D_{-x} e_{h}^{n}\right)_{h,+} \cdot
$$

If follows from (36) that for $Z_{2,1}^{n}$ holds the following

$$
\begin{aligned}
\left|Z_{2,1}^{n}\right| \leq h_{\max }^{4} C_{Z_{2}}\left(\frac{a_{b}^{2}}{4 \varepsilon_{4}^{2}}\|c\|_{\mathscr{C}\left([0, T], H^{3}(0,1)\right)}^{2}+\frac{\left(a_{b}^{\prime}\right)^{2}}{4 \varepsilon_{5}^{2}}\|c\|_{\mathscr{C}\left([0, T], W^{1, \infty}(0,1)\right)}^{2}\|c\|_{\mathscr{C}\left([0, T], H^{2}(0,1)\right)}^{2}\right) \\
+\left(\varepsilon_{4}^{2}+\varepsilon_{5}^{2}\right)\left\|D_{-x} e_{h}^{n}\right\|_{h,+}^{2}
\end{aligned}
$$

where $\varepsilon_{4}, \varepsilon_{5} \neq 0$.

For $Z_{2,2}^{n}$ it can be shown that

$$
\left|Z_{2,2}^{n}\right| \leq \Delta t \frac{\left(a_{b}^{\prime}\right)^{2}}{4 \varepsilon_{6}^{2}}\left\|R_{h} c\right\|_{H^{1}\left(t_{n-1}, t_{n}, \mathbb{W}_{h}\right)}^{2}\|c\|_{\mathscr{C}\left([0, T], W^{1, \infty}(0,1)\right)}^{2}+\varepsilon_{6}^{2}\left\|D_{-x} e_{h}^{n}\right\|_{h,+}^{2},
$$

where $\varepsilon_{6} \neq 0$.

- Estimate for $Z_{3}^{n}$ : We remark that $Z_{3}^{n}$ admits the decomposition

$$
Z_{3}^{n}=Z_{3,1}^{n}+Z_{3,2}^{n}
$$

with

$$
Z_{3,1}^{n}=\int_{0}^{t_{n}} k_{e r}\left(t_{n}-s\right)\left(d\left(M_{h} c(s)\right) D_{-x} R_{h} c(s)-\left(d\left(\hat{M}_{h} c(s)\right) \hat{M}_{h} \frac{\partial c}{\partial x}(s), D_{-x} e_{h}^{n}\right)_{h,+} d s\right.
$$

and

$$
\begin{aligned}
Z_{3,2}^{n} & =\Delta t \sum_{\ell=0}^{n-1} k_{e r}\left(t_{n}-t_{\ell}\right)\left(d\left(M_{h} c\left(t_{\ell}\right)\right) D_{-x} R_{h} c\left(t_{\ell}\right), D_{-x} e_{h}^{n}\right)_{h,+} \\
& -\int_{0}^{t_{n}} k_{e r}\left(t_{n}-s\right)\left(d\left(M_{h} c(s)\right) D_{-x} R_{h} c(s), D_{-x} e_{h}^{n}\right)_{h,+} d s .
\end{aligned}
$$


Following the proof of the estimate (38), it can be shown that for $Z_{3,1}^{n}$ we have

$$
\begin{aligned}
\left|Z_{3,1}^{n}\right| \leq & h_{\max }^{4} C_{Z_{3}} \frac{1}{4 \varepsilon_{7}^{2}} k\left(d_{b}^{2}+\left(d_{b}^{\prime}\right)^{2}\|c\|_{\mathscr{C}\left([0, T], W^{1, \infty}(0,1)\right)}^{2}\right)\|c\|_{L^{2}\left(0, T, H^{3}(0,1)\right)}^{2} \\
& +2 \varepsilon_{7}^{2}\left\|D_{-x} e_{h}^{n}\right\|_{h,+}^{2}
\end{aligned}
$$

with $\varepsilon_{7} \neq 0$.

To obtain an estimate for $Z_{3,2}^{n}$ we remark that this term represents the error of the rectangular rule.Then

$$
\begin{aligned}
\left|Z_{3,2}^{n}\right| \leq & \Delta t d_{b} \int_{0}^{T}\left|k_{e r}^{\prime}\left(t_{n}-s\right)\right|\|c(s)\|_{W^{1, \infty}(0,1)}\left\|D_{-x} e_{h}^{n}\right\|_{h,+} \\
& +\Delta t d_{b}^{\prime} \int_{0}^{T}\left|k_{e r}\left(t_{n}-s\right)\right|\left\|R_{h} \frac{d c}{d t}(s)\right\|\left\|_{h}\right\| c(s)\left\|_{W^{1, \infty}(0,1)}\right\| D_{-x} e_{h}^{n} \|_{h,+} \\
& +\Delta t d_{b} \int_{0}^{T}\left|k_{e r}\left(t_{n}-s\right)\right|\left\|\frac{d c}{d t}(s)\right\|_{W^{1, \infty}(0,1)}\left\|D_{-x} e_{h}^{n}\right\|_{h,+}
\end{aligned}
$$

that leads to

$$
\begin{aligned}
\left|Z_{3,2}^{n}\right| \leq \Delta t^{2} k & \frac{1}{4 \varepsilon_{8}^{2}}\left(d_{b}^{2}\left(\|c\|_{L^{2}\left(0, T, W^{1, \infty}(0,1)\right)}^{2}+\|c\|_{H^{1}\left(0, T, W^{1, \infty}(0,1)\right)}^{2}\right)\right. \\
& \left.+\left(d_{b}^{\prime}\right)^{2}\|c\|_{\mathscr{C}\left([0, T], W^{1, \infty}(0,1)\right)}^{2}\left\|R_{h} c\right\|_{H^{1}\left(0, T, \mathbb{W}_{h}\right)}^{2}\right)+3 \varepsilon_{8}\left\|D_{-x} e_{h}^{n}\right\|_{h,+}^{2}
\end{aligned}
$$

where $\varepsilon_{8} \neq 0$.

Considering in (61) the obtained estimates with $\varepsilon_{i}=\varepsilon, i=0, \ldots, 8$, we deduce

$$
\begin{gathered}
\left\|e_{h}^{n}\right\|_{h}^{2}+2\left(a_{0}-12 \varepsilon^{2}\right) \Delta t\left\|D_{-x} e_{h}^{n}\right\|_{h,+}^{2} \leq(1+\Delta t \Phi)\left\|e_{h}^{n-1}\right\|_{h}^{2}+2 \varepsilon^{2} \Delta t\left\|e_{h}^{n}\right\|_{h}^{2} \\
+\Delta t^{2} \Psi \sum_{\ell=0}^{n-1}\left\|D_{-x} e_{h}^{\ell}\right\|_{h,+}^{2}+\Delta t T_{h}^{n},
\end{gathered}
$$

where $\Phi$ and $\Psi$ are defined by (59) and (60), respectively, and $T_{h}^{n}$ is given by (56).

From inequality (74) we deduce

$$
\begin{gathered}
\left(1-2 \varepsilon^{2} \Delta t\right)\left\|e_{h}^{n}\right\|_{h}^{2}+2\left(a_{0}-12 \varepsilon^{2}\right) \Delta t \sum_{j=1}^{n}\left\|D_{-x} e_{h}^{j}\right\|_{h,+}^{2} \leq\left\|e_{h}^{0}\right\|_{h}^{2}+\left(\Phi+2 \varepsilon^{2}\right) \Delta t \sum_{j=0}^{n-1}\left\|e_{h}^{j}\right\|_{h}^{2} \\
+\Delta t^{2} \Psi \sum_{j=1}^{n} \sum_{\ell=0}^{j-1}\left\|D_{-x} e_{h}^{\ell}\right\|_{h,+}^{2}+\Delta t \sum_{\ell=1}^{n} T_{h}^{\ell}
\end{gathered}
$$

that can be rewritten in the following equivalent form

$$
\begin{aligned}
& \left\|e_{h}^{n}\right\|_{h}^{2}+\Delta t \sum_{\ell=0}^{n}\left\|D_{-x} e_{h}^{\ell}\right\|_{h,+}^{2} \leq \\
& \frac{\max \left\{\Phi+2 \varepsilon^{2}, \Psi\right\} \Delta t}{\min \left\{1-\Delta t 2 \varepsilon^{2}, 2\left(a_{0}-12 \varepsilon^{2}\right)\right\}} \sum_{j=0}^{n-1}\left(\left\|e_{h}^{j}\right\|_{h}^{2}+\Delta t \sum_{\ell=0}^{j}\left\|D_{-x} e_{h}^{\ell}\right\|_{h,+}^{2}\right) \\
& +\frac{1}{\min \left\{1-\Delta t 2 \varepsilon^{2}, 2\left(a_{0}-12 \varepsilon^{2}\right)\right\}}\left(\left\|e_{h}^{0}\right\|_{h,+}^{2}+2\left(a_{0}-12 \varepsilon^{2}\right) \Delta t\left\|D_{-x} e_{h}^{0}\right\|_{h,+}^{2}+\Delta t \sum_{\ell=1}^{n} T_{h}^{\ell}\right),
\end{aligned}
$$


for $n=1, \ldots, M$, provided that (57) and (58) hold.

Applying a discrete Gronwall lemma, we obtain (55).

We remark that conditions (42) and (58) define un upper bound for $\Delta t$ that depends on the inverse of the lower bound $a_{0}$ for the diffusion coefficient.

As a corollary of Theorem 2 we have the next convergence result.

Corollary 1. Let $c \in \mathscr{C}\left([0, T], H^{3}(0,1) \cap H_{0}^{1}(0,1)\right) \cap \mathscr{C}^{1}\left([0, T], H^{2}(0,1)\right)$ be the solution of $(\mathbf{V P})$ and let $c_{h}^{n}$ be its approximation defined by (50). Under the conditions of Theorem 2, there exists positive constant $C_{e r}$ that does not depend on $h, \Delta t$ neither $c$, such that $e_{h}^{n}=R_{h} c\left(t_{n}\right)-c_{h}^{n}, n=0, \ldots, M$, satisfies

$$
\left\|e_{h}^{n}\right\|_{h}^{2}+\Delta t \sum_{\ell=0}^{n}\left\|D_{-x} e_{h}^{\ell}\right\|_{h,+}^{2} \leq C_{e r}\left(h_{\max }^{4}+\Delta t^{2}\right)
$$

provided that $\Delta$ t satisfies (58).

\subsection{Weakly singular kernels}

In the previous section the convergence analysis of the IMEX method (50) (or (53)) was established in Corollary 1 under the assumptions (54) for the kernel $k_{e r}$. As in the semi-discrete case, in what follows we establish a new error estimates considering weaker conditions on $k_{e r}$. In order to do that we replace (50) by

$$
\begin{aligned}
& D_{-t} c_{h}^{n}\left(x_{j}\right)=D_{x}^{*}\left(a\left(M_{h} c_{h}^{n-1}\left(x_{j}\right)\right) D_{-x} c_{h}^{n}\left(x_{j}\right)\right)+f\left(x_{j}, t_{n}\right) \\
& +\sum_{\ell=0}^{n-1} \int_{t_{\ell}}^{t_{\ell+1}} k_{e r}\left(t_{n}-s\right) d s D_{x}^{*}\left(d\left(M_{h} c_{h}^{\ell}\left(x_{j}\right)\right) D_{-x} c_{h}^{\ell}\left(x_{j}\right)\right), \\
& j=1, \ldots, N-1 \text {, }
\end{aligned}
$$

that is equivalent to

$$
\begin{array}{r}
\left(D_{-t} c_{h}^{n}, w_{h}\right)_{h}=-\left(a\left(M_{h} c_{h}^{n-1}\right) D_{-x} c_{h}^{n}, D_{-x} w_{h}\right)_{h,+}+\left(f_{h}\left(t_{n}\right), w_{h}\right)_{h} \\
-\sum_{\ell=0}^{n-1} \int_{t_{\ell}}^{t_{\ell+1}} k_{e r}\left(t_{n}-s\right) d s\left(d\left(M_{h} c_{h}^{\ell}\right) D_{-x} c_{h}^{\ell}, D_{-x} w_{h}\right)_{h,+}, n=1, \ldots, M,
\end{array}
$$

for all $w_{h} \in \mathbb{W}_{h, 0}$.

In the convergence analysis of method (50) (or (51)) it was assumed conditions (54) for the kernel $k_{e r}$. Following the proof of Theorem 2, for $e_{h}^{n}=R_{h} c\left(t_{n}\right)-c_{h}^{n}, n=0, \ldots, M$, holds (61) where $T_{i n t, d}^{n}$ and $Z_{3}^{n}$ are given by

$$
\begin{aligned}
& T_{i n t, d}^{n}= \\
& \sum_{\ell=0}^{n-1} \int_{t_{\ell}}^{t_{\ell+1}} k_{e r}\left(t_{n}-s\right) d s\left(\left(d\left(M_{h} c_{h}^{\ell}\right) D_{-x} c_{h}^{\ell}, D_{-x} e_{h}^{n}\right)_{h,+}-\left(d\left(M_{h} c\left(t_{\ell}\right)\right) D_{-x} R_{h} c\left(t_{\ell}\right), D_{-x} e_{h}^{n}\right)_{h,+}\right), \\
& Z_{3}^{n}=\sum_{\ell=0}^{n-1} \int_{t_{\ell}}^{t_{\ell+1}} k_{e r}\left(t_{n}-s\right)\left(\left(d\left(M_{h} c\left(t_{\ell}\right)\right) D_{-x} R_{h} c\left(t_{\ell}\right)-d\left(\hat{M}_{h} c(s)\right) \hat{M}_{h} \frac{\partial c}{\partial x}(s), D_{-x} e_{h}^{n}\right)_{h,+}\right) d s
\end{aligned}
$$


and $T_{a}^{n}, Z_{1}^{n}$ and $Z_{2}^{n}$, are given by (62), (63) and (64), respectively. For $T_{a}^{n}, Z_{1}^{n}$ the estimates (65) and (67), hold, respectively. The error term $Z_{2}^{n}$ is decomposed into $Z_{2,1}^{n}+Z_{2,2}^{n}$ where $Z_{2,1}^{n}$ and $Z_{2,2}^{n}$ are defined by (68) and (69), respectively. It can be shown that for $Z_{2,1}^{n}$ and $Z_{2,2}^{n}$ the estimates (67) and (71) hold. We estimate now $T_{i n t, d}^{n}$ and $Z_{3}^{n}$.

- Estimate for $T_{i n t, d}^{n}$ : It can be shown that

$$
\begin{aligned}
\left|T_{i n t, d}^{n}\right| \leq & \frac{1}{4 \varepsilon_{1}^{2}} k\left(d_{b}^{2}+\left(d_{b}^{\prime}\right)^{2}\|c\|_{\mathscr{C}\left([0, T], W^{1, \infty}(0,1)\right)}^{2}\right) \sum_{\ell=0}^{n-1} \int_{t_{\ell}}^{t_{\ell+1}}\left|k_{e r}\left(t_{n}-s\right)\right| d s\left\|D_{-x} e_{h}^{\ell}\right\|_{h,+}^{2} \\
& +2 \varepsilon_{1}^{2}\left\|D_{-x} e_{h}^{n}\right\|_{h,+}^{2}
\end{aligned}
$$

where $\varepsilon_{1} \neq 0$ and $k=\left\|k_{e r}\right\|_{L^{1}(0, T)}$.

- Estimate for $Z_{3}^{n}$ : Let $Z_{3,1}^{n}$ be defined by (72) and $Z_{3,2}^{n}$ be defined by

$$
\begin{aligned}
& Z_{3,2}^{n}= \\
& \sum_{\ell=0}^{n-1} \int_{t_{\ell}}^{t_{\ell+1}} k_{e r}\left(t_{n}-s\right)\left(d\left(M_{h} c\left(t_{\ell}\right)\right) D_{-x} R_{h} c\left(t_{\ell}\right)-d\left(M_{h} c(s)\right) D_{-x} R_{h} c(s), D_{-x} e_{h}^{n}\right)_{h,+} d s .
\end{aligned}
$$

We have $Z_{3}^{n}=Z_{3,1}^{n}+Z_{3,2}^{n}$ where

$$
\begin{aligned}
\left|Z_{3,1}^{n}\right| \leq & h_{\max }^{4} \frac{C_{Z_{3}} k}{4 \varepsilon_{7}^{2}}\left(d_{b}^{2}+\left(d_{b}^{\prime}\right)^{2}\|c\|_{\mathscr{C}\left([0, T], W^{1, \infty}(0,1)\right)}^{2}\right) \int_{0}^{t_{n}}\left|k_{e r}\left(t_{n}-s\right)\right| d s\|c\|_{\mathscr{C}\left([0, T] H^{3}(0,1)\right)}^{2} \\
& +2 \varepsilon_{7}^{2}\left\|D_{-x} e_{h}^{n}\right\|_{h,+}^{2}
\end{aligned}
$$

with $\varepsilon_{7} \neq 0$ and

$$
\begin{aligned}
\left|Z_{3,2}^{n}\right| \leq & \frac{1}{4 \varepsilon_{8}^{2}} k \Delta t \sum_{\ell=0}^{n-1} \int_{t_{\ell}}^{t_{\ell+1}}\left|k_{e r}\left(t_{n}-s\right)\right| d s\left(\left(d_{b}^{\prime}\right)^{2}\|c\|_{\mathscr{C}\left([0, T], W^{1, \infty}(0,1)\right)}^{2}\left\|R_{h} c\right\|_{H^{1}\left(\left[t_{\ell}, t_{\ell+1}\right], \mathbb{W}_{h}\right)}^{2}\right. \\
& \left.+d_{b}^{2}\|c\|_{H^{1}\left(\left[t_{\ell}, t_{\ell+1}\right], W^{1, \infty}(0,1)\right)}^{2}\right)+2 \varepsilon_{8}^{2}\left\|D_{-x} e_{h}^{n}\right\|_{h,+}^{2}
\end{aligned}
$$

with $\varepsilon_{8} \neq 0$.

As in the proof of Theorem 2, it can be shown that $e_{h}^{n}$ satisfies

$$
\begin{gathered}
\left(1-2 \varepsilon^{2} \Delta t\right)\left\|e_{h}^{n}\right\|_{h}^{2}+2\left(a_{0}-11 \varepsilon^{2}\right) \Delta t \sum_{j=1}^{n}\left\|D_{-x} e_{h}^{j}\right\|_{h,+}^{2} \leq\left\|e_{h}^{0}\right\|_{h}^{2}+\left(\Phi+2 \varepsilon^{2}\right) \Delta t \sum_{j=0}^{n-1}\left\|e_{h}^{j}\right\|_{h}^{2} \\
+\Delta t \Psi \sum_{j=1}^{n} \sum_{\ell=0}^{j-1} \int_{t_{\ell}}^{t_{\ell+1}}\left|k_{e r}\left(t_{j}-s\right)\right| d s\left\|D_{-x} e_{h}^{\ell}\right\|_{h,+}^{2}+\Delta t \sum_{j=1}^{n} T_{h}^{j}
\end{gathered}
$$


where $\Phi$ and $\Psi$ are defined by (59) and (60), respectively, with $k=\left\|k_{e r}\right\|_{L^{1}(0, T)}$ and $T_{h}^{\ell}$ is given now by

$$
\begin{aligned}
& T_{h}^{j}=C_{T} \frac{1}{2 \varepsilon^{2}} \quad\left(\Delta t \left(\left\|R_{h} c\right\|_{H^{2}\left(t_{j-1}, t_{j}, \mathbb{W}_{h}\right)}^{2}+a_{b}^{2}\left\|R_{h} c\right\|_{H^{1}\left(t_{j-1}, t_{j}, \mathbb{W}_{h}\right)}^{2}\|c\|_{\mathscr{C}\left([0, T], W^{1, \infty}(0,1)\right)}^{2}\right.\right. \\
& +k \sum_{\ell=0}^{j-1} \int_{t_{\ell}}^{t_{\ell+1}}\left|k_{e r}\left(t_{j}-s\right)\right| d s\left(d_{b}^{2}\|c\|_{H^{1}\left(\left[t_{\ell}, t_{\ell+1}\right], W^{1, \infty}(0,1)\right)}^{2}\right. \\
& \left.\left.+\left(d_{b}^{\prime}\right)^{2}\|c\|_{\mathscr{C}\left([0, T], W^{1, \infty}(0,1)\right)}^{2}\left\|R_{h} c\right\|_{H^{1}\left(\left[t_{\ell}, t_{\ell+1}\right], \mathbb{W}_{h}\right)}^{2}\right)\right) \\
& +h_{\max }^{4}\left(\|c\|_{\mathscr{C}\left([0, T], H^{2}(0,1)\right)}^{2}+\left(a_{b}^{2}+\left(a_{b}^{\prime}\right)^{2}\|c\|_{\mathscr{C}\left([0, T], W^{1, \infty}(0,1)\right)}^{2}\right)\|c\|_{\mathscr{C}\left([0, T], H^{3}(0,1)\right)}^{2}\right. \\
& \left.\left.+k^{2}\left(d_{b}^{2}+\left(d_{b}^{\prime}\right)^{2}\|c\|_{\mathscr{C}\left([0, T], W^{1, \infty}(0,1)\right)}^{2}\right)\|c\|_{\mathscr{C}\left([0, T], H^{3}(0,1)\right)}^{2}\right)\right)
\end{aligned}
$$

As for $A=\sum_{j=1}^{n} \sum_{\ell=0}^{j-1} \int_{t_{\ell}}^{t_{\ell+1}}\left|k_{e r}\left(t_{j}-s\right)\right| d s\left\|D_{-x} e_{h}^{\ell}\right\|_{h,+}^{2}$ we have successively the following

$$
\begin{aligned}
& A=\sum_{\ell=0}^{n-1} \sum_{j=\ell+1}^{n} \int_{t_{\ell}}^{t_{\ell+1}}\left|k_{e r}\left(t_{j}-s\right)\right| d s\left\|D_{-x} e_{h}^{\ell}\right\|_{h,+}^{2} \\
& =\quad \sum_{\ell=0}^{n-1} \sum_{j=\ell+1}^{n} \int_{(j-\ell-1) \Delta t}^{(j-\ell) \Delta t}\left|k_{e r}(s)\right| d s\left\|D_{-x} e_{h}^{\ell}\right\|_{h,+}^{2} \\
& \leq \quad\left\|k_{e r}\right\|_{L^{1}(0, T)} \sum_{\ell=0}^{n}\left\|D_{-x} e_{h}^{\ell}\right\|_{h,+}^{2},
\end{aligned}
$$

from (82) we deduce

$$
\begin{gathered}
\left(1-2 \varepsilon^{2} \Delta t\right)\left\|e_{h}^{n}\right\|_{h}^{2}+\quad \\
\quad\left(2\left(a_{0}-11 \varepsilon^{2}\right)-k \Psi\right) \Delta t \sum_{j=0}^{n}\left\|D_{-x} e_{h}^{j}\right\|_{h,+}^{2} \leq\left(\Phi+2 \varepsilon^{2}\right) \Delta t \sum_{j=0}^{n-1}\left\|e_{h}^{j}\right\|_{h}^{2} \\
+\left\|e_{h}^{0}\right\|_{h}^{2}+2\left(a_{0}-11 \varepsilon^{2}\right) \Delta t\left\|D_{-x} e_{h}^{0}\right\|_{h,+}^{2}+\Delta t \sum_{j=1}^{n} T_{h}^{j} .
\end{gathered}
$$

If $\varepsilon$ and $\Delta t$ are such that (58) holds and

$$
2\left(a_{0}-11 \varepsilon^{2}\right)-k \Psi>0,
$$

then

$$
\begin{aligned}
& \left\|e_{h}^{n}\right\|_{h}^{2}+\Delta t \sum_{j=0}^{n}\left\|D_{-x} e_{h}^{j}\right\|_{h,+}^{2} \leq \\
& \frac{e^{\left(\Phi+2 \varepsilon^{2}\right) T}}{\min \left\{1-2 \varepsilon^{2} \Delta t, 2\left(a_{0}-11 \varepsilon^{2}\right)-k \Psi\right\}}\left(\left\|e_{h}^{0}\right\|_{h}^{2}+2\left(a_{0}-11 \varepsilon^{2}\right) \Delta t\left\|D_{-x} e_{h}^{0}\right\|_{h,+}^{2}+\Delta t \sum_{j=1}^{n} T_{h}^{j}\right) .
\end{aligned}
$$

The upper bound (86) is established under the condition $k_{e r} \in L^{1}(0, T)$. This upper bound allows us to conclude that for the the error $e_{h}^{n}$ induced by the method (78) estimate (76) holds. While the kernel function presents lower smoothness, the set of conditions on the time step size (58) and (85) are more severe than those imposed for non singular kernels. 


\section{Numerical Simulations}

The aim of this section is to illustrate the convergence results obtained in the paper. We start by considering the IMEX method (50) (or (53)) when $k_{e r}$ is a smooth function in the sense that it satisfies condition (54).

Let us consider in (1)-(4)

$$
a(c)=1+c, d(c)=10 c, k_{e r}=e^{-\frac{1}{2} t},
$$

and $f$, the initial and boundary conditions selected such that this IBVP has the following solution

$$
c(x, t)=e^{-t}(1-x)(\arctan (\alpha(x-\bar{x}))+\arctan (\alpha \bar{x})), x \in[0,1], t \in[0, T],
$$

where $\bar{x} \in(0,1)$. For large values of $\alpha, c$ has an interior-layer in the neighborhood of $x=\bar{x}$ (see [8]).

The numerical approximation $c_{h}$ was obtained with the method (50)-(52) when we consider $\bar{x}=\frac{1}{2}, \alpha=80$, with nonuniform grids in the spatial domain and with an uniform grid in the time domain with $T=0.1$ and $\Delta t=1 \times 10^{-7}$. The initial spatial grid $I_{h}$ was arbitrary and the following grids $I_{h}$ were obtained introducing in $\left[x_{j}, x_{j+1}\right]$ the midpoint.

In Table 1 we present the error

$$
\mathbb{E}_{p}=\max _{n}\left(\left\|e_{h}\left(t_{n}\right)\right\|_{h_{p}}^{2}+\Delta t \sum_{s=1}^{n}\left\|e_{h}(s)\right\|_{1, h_{p}}^{2}\right)^{\frac{1}{2}},
$$

and the rate $R_{p}$ defined by

$$
R_{p}=\frac{\ln \left(\mathbb{E}_{p} / \mathbb{E}_{p+1}\right)}{\ln \left(h_{p_{\max }} / h_{p+1_{\max }}\right)} .
$$

\begin{tabular}{|l|l|l|c|}
\hline$N_{p}$ & $h_{p_{\max }}$ & $\mathbb{E}_{p}$ & $R_{p}$ \\
\hline 34 & $4.2514 \times 10^{-2}$ & $2.4837 \times 10^{-2}$ & - \\
\hline 68 & $2.1257 \times 10^{-2}$ & $6.5927 \times 10^{-3}$ & 1.9136 \\
\hline 136 & $1.0628 \times 10^{-2}$ & $1.6906 \times 10^{-3}$ & 1.9633 \\
\hline 272 & $5.3142 \times 10^{-3}$ & $4.2602 \times 10^{-4}$ & 1.9886 \\
\hline 544 & $2.6571 \times 10^{-3}$ & $1.0610 \times 10^{-4}$ & 2.0055 \\
\hline 1088 & $1.3286 \times 10^{-3}$ & $2.6492 \times 10^{-5}$ & 2.0017 \\
\hline 2176 & $6.6428 \times 10^{-4}$ & $6.6132 \times 10^{-6}$ & 2.0021 \\
\hline 4352 & $3.3214 \times 10^{-4}$ & $1.6449 \times 10^{-6}$ & 2.0074 \\
\hline
\end{tabular}

Table 1: Convergence order non singular kernels.

We note that the numerical results presented in Table 1 agree with the theoretical results presented in Theorem 1 and Corollary 1 that is $\mathbb{E}_{p}=O\left(h_{\text {max }}^{2}\right)$. 
Let us consider now the IMEX method (77) (or (78)) studied when $k_{e r} \in L^{1}(0, T)$. In (1)-(4) we consider

$$
a(c)=10+c, d(c)=2, k_{e r}=\frac{1}{\sqrt{t}},
$$

and $f$, the initial and boundary conditions selected such that this IBVP has the following solution

$$
c(x, t)=t^{2}(1-x)(\arctan (\alpha(x-\bar{x}))+\arctan (\alpha \bar{x})), x \in[0,1], t \in[0, T],
$$

where $\bar{x} \in(0,1)$.

Let $\alpha=80$ and $\bar{x}=\frac{1}{2}$, in Table 2 we present the error $\mathbb{E}_{p}$ and the convergence rate $R_{p}$ defined respectively by (89) and (90). We observe that in agreement with (86) we have that $\mathbb{E}_{p}=O\left(h_{\max }^{2}\right)$.

\begin{tabular}{|l|l|l|c|}
\hline$N_{p}$ & $h_{p_{\max }}$ & $\mathbb{E}_{p}$ & $R_{p}$ \\
\hline 48 & $3.2407 \times 10^{-2}$ & $1.6811 \times 10^{-2}$ & - \\
\hline 96 & $1.6204 \times 10^{-2}$ & $4.8871 \times 10^{-3}$ & 1.7823 \\
\hline 192 & $8.1019 \times 10^{-3}$ & $1.2970 \times 10^{-3}$ & 1.9138 \\
\hline 384 & $4.0509 \times 10^{-3}$ & $3.3723 \times 10^{-4}$ & 1.9434 \\
\hline 768 & $2.0255 \times 10^{-3}$ & $8.4680 \times 10^{-5}$ & 1.9936 \\
\hline 1536 & $1.0127 \times 10^{-3}$ & $2.1257 \times 10^{-5}$ & 1.9941 \\
\hline 3072 & $5.0637 \times 10^{-4}$ & $5.2172 \times 10^{-6}$ & 2.0266 \\
\hline
\end{tabular}

Table 2: Convergence order weakly singular kernels.

\section{Conclusions}

In this paper we propose a finite difference method to solve numerically the IBVP defined by the quasilinear integro-differential equation (1) of Volterra type with Dirichlet boundary conditions. We point out that the non Fickian equation (1) can be used, as previously mentioned, to model a large number of physical situations where Fick's law is not appropriate to describe the mass flux and a delay effect is needed. The finite difference method (17) can be seen as a fully discrete in space piecewise linear finite element method. Methods of this class were studied for elliptic equations for instance in [4] , [15], [16] and [21].

In the main theorems of this paper - Theorem 1 and Theorem 2, we prove that a discrete $L^{2}$ norm of the spatial discretization error and of its discrete gradient are second order convergent with respect to space step size while the spatial truncation error is only of first order with respect to infinity norm for non singular kernels that satisfy conditions (54). The version of these results for kernels $k_{e r} \in L^{1}(0, T)$ are also established. The approach used to prove these results was introduced in [24] for a linear version of (17) and differs from the one usually followed in the literature and which was introduced by Wheeler in [37]. Our approach allows, for the semi-discrete and fully discrete approximations the weakening 
of the smoothness conditions usually required when Wheeler's technique is used. In fact, for instance for the semi-discrete approximation we replace $c \in H^{1}\left(0, T, H^{3}(0,1)\right) \cap L^{2}\left(0, T, H^{3}(0,1) \cap H_{0}^{1}(0,1)\right)$ by $c \in H^{1}\left(0, T, H^{2}(0,1)\right) \cap L^{2}\left(0, T, H^{3}(0,1) \cap H_{0}^{1}(0,1)\right)$.

For the sake of simplicity only the one dimensional case was studied, but the techniques here presented can be used to extend the analysis for two dimensional problems.

\section{Acknowledgements}

This work was partially supported by Centro de Matemática da Universidade de Coimbra (CMUC), funded by the European Regional Development Fund through the program COMPETE and by the Portuguese Government through the FCT - Fundação para a Ciência e Tecnologia under the projects PEstC/MAT/UI0324/2011 and UTAustin/MAT/0066/2008. The authors also thank the referees for their helpful comments and suggestions, which contributed to the improvement of the obtained results.

\section{References}

[1] A. Araújo, J. A. Ferreira and P. de Oliveira, The effect of memory terms in diffusion phenomena, J. Comput. Math., 24 (2006), pp. 91-102.

[2] A. Araújo, J. A. Ferreira and P. de Oliveira, Qualitative behaviour of numerical traveling waves solutions for reaction diffusion equations with memory, Appl. Anal., 84 (2005), pp. 1231-1246.

[3] A. A. Bojeldain, Existence and uniqueness theorems for a class of nonlinear Volterra integrodifferential equations, Ann. Univ. Sci. Budap., Sect. Comput, 15 (1995), pp. 143-156.

[4] S. Barbeiro, J. A. Ferreira and R. Grigorieff, Supraconvergence of a finite difference scheme for solutions in $H^{s}(0, L)$, IMA J. Numer. Anal., 25 (2005), pp. 797-811.

[5] S. Barbeiro, J. A. Ferreira and L. Pinto, $H^{1}$-second order convergent estimates for non Fickian models, Appl. Numer. Math., 61 (2011), pp. 201-215.

[6] J. H. Bramble and S. R. Hilbert, Estimation of linear functionals on Sobolev spaces with application to Fourier transforms and spline interpolation, SIAM J. Numer. Anal., 7 (1970), pp. 112-124.

[7] H. F. Brinson and L. C. Brinson, Polymer Engineering Science and Viscoelasticity - An Introduction, Springer, New York, 2008.

[8] G. F. Carey and D. L. Humphrey, Finite element mesh refinement algorithm using element residuals, Lect. Notes Comput. Sci., 76 (1979), pp. 243-249.

[9] C. Chunmiao and S. Tsimin, Finite Element Methods for Integro Differential Equations, World Scientific Publishers, Singapore, 1998.

[10] J. R. Branco, J. A. Ferreira and P. de Oliveira, Numerical methods for the generalized FisherKolmogorov-Petrovskii-Piskunov equation, Appl. Numer. Math., 57 (2007), pp. 89-102.

[11] D. A. Edwards and D. S. Cohen, An unusual moving boundary condition arising in anomalous diffusion problems, SIAM J. Appl. Math., 55 (1995), pp. 662-676. 
[12] D. A. Edwards and D. S. Cohen, A mathematical model for a dissolving polymer, AIChE J., 41 (1995), pp. 2345-2355.

[13] D. A. Edwards, Non-Fickian Diffusion in thin polymer films, J. Polym. Sci., Part B: Polym. Phys., 34 (1996), pp. 981-997.

[14] D. A. Edwards, A spatially nonlocal model for polymer-penetrant diffusion, J. Appl. Math. Phys., 52 (2001), pp. 254-288.

[15] E. Emmrich, Supraconvergence and supercloseness of a discretization for elliptic third kind boundary value problems on polygonal domains, Comput. Methods Appl. Math., 7 (2007), pp. 153-162.

[16] E. Emmrich, R. D. Grigorieff, Supraconvergence of a finite difference scheme for elliptic boundary value problems of the third kind in fractional order Sobolev spaces, Comput. Methods Appl. Math., 6, (2006) 154-177.

[17] S. Fedotov, Traveling waves in a reaction-diffusion system: Diffusion with finite velocity and Kolmogorov-Petrovskii-Piskunov kinetics, Phys. Rev. E: Stat. Phys., Plasmas, Fluids, 58 (1998): pp. 5143-5145.

[18] S. Fedotov, Nonuniform reaction rate distribution for the generalized Fisher equation: Ignition ahead of the reaction front, Phys. Rev. E: Stat. Phys., Plasmas, Fluids, 60 (1999), 4958-4961.

[19] S. Fedotov and A. Iomin, Migration and proliferation dichotomy in tumor-cell invasion, Phys. Rev. Lett., 98 (2007), pp. 118101(1)-(4).

[20] S. Fedotov and A. Iomin, Probabilistic approach to a proliferation and migration dichotomy in tumor cell invasion, Phys. Rev. E: Stat. Phys., Plasmas, Fluids, 77 (2008), pp. 1031911(1)-(10).

[21] J. A. Ferreira and R. D. Grigorieff, Supraconvergence and supercloseness of a scheme for elliptic equations on nonuniform grids, Numer. Funct. Anal. Optim., 27 (2006), pp. 539-564.

[22] J. A. Ferreira and P. de Oliveira, Looking for a lost memory in diffusion-reaction equations, in Advances in Mathematical Fluid Mechanic, (R. Ranacker and A. Sequeira, eds.), Springer-Verlag Berlin Heidelberg, 2010, pp. 229-252.

[23] J. A. Ferreira, P. de Oliveira, P. da Silva, Reaction-diffusion in viscoelastic materials, J. Comput. Appl. Math., 236 (2012), pp. 3783-3795.

[24] J. A. Fereira, L. Pinto and G. Romanazzi, Supraconvergence and Supercloseness in Volterra equations, Appl. Numer. Math., 62 (2012), pp. 1718-1739.

[25] G. Franceschini, The mechanics of humain brain tissue, PhD Thesis, University of Trento, 2006.

[26] M. Grasselli and A. Lorenzi, Abstract nonlinear Volterra integrodifferential equations with nonsmooth kernels, Atti Accad. Naz. Lincei, Cl. Sci. Fis. Mat. Nat., Rend. Lincei, Mat. Appl., 2 (1991), No. 1, pp. 43-53.

[27] M. Grassi and G. Grassi, Mathematical modelling and controlled drug delivery : matrix systems, Curr. Drug Deliv., 2 (2005), pp. 97-116. 
[28] S. M. Hassahizadeh, On the transient non-Fickian dispersion theory, Transp. Porous Media, 23 (1996), pp. 107-124.

[29] J. D. Humphrey, Continuum biomechanics of soft biological tissues, Lond. R. S. Proc., 459 (2003), pp. 3-46.

[30] H-M Yin, Weak and classical solutions of some nonlinear Volterra integrodifferential equations, Commun. Partial Differ. Equations, 17 (1992), pp. 1369-1385.

[31] P. M. D. Khan and B. G. Pachpatte, On quasilinear Volterra integrodifferential equations in Banach spaces, Indian J. Pure Appl. Math., 18 (1987), pp. 32-49.

[32] C. Maas, A hyperbolic dispersion equation to model the bounds of a contaminated groundwater body, J. Hydrology, 226 (1999), pp. 234-241.

[33] W. McLean, V. Thoméeb and L.B. Wahlbinc, Discretization with variable time steps of an evolution equation with a positive-type memory term, J. Comput. Appl. Math., 69 (1996), pp. 49-69.

[34] W. McLean and V. Thomée, Numerical solution of an evolution equation with a positive-type memory term, J. Aust. Math. Soc. Appl. Math., 35 (1993), pp. 23-70.

[35] A. Mehrabian and Y. Abousleiman, A general solution to a poroviscoelastic model of hydrocephalic humain brain tissue, J. Theor. Biol., 29 (2011), pp. 105-118.

[36] S. P. Neuman and D. M. Tartakovski, Perspectives on theories of non-Fickian transport in heterogeneous medias, Adv. Water Resour., 32 (2008), pp. 670-680.

[37] M. F. Wheeler, A priori $L^{2}$ error estimates for Galerkin approximation to parabolic partial differential equations, SIAM J. Numer. Anal., 10 (1973), pp. 723-759.

[38] S. Shaw and J. R. Whiteman, Some partial differential Volterra equation problems arising in viscoelasticity, in: Proceedings of the Conference on Differential Equations and their Applications, Brno, August 25-29, 1997, (R. P. Agarwal, F. Neuman and J. Vosmanský, eds.), Masaryk University, Brno, 1998, pp. 183-200. 